\title{
Soft Actuators and Robotic Devices for Rehabilitation and Assistance
}

\author{
Min Pan ${ }^{1}$, Chenggang Yuan ${ }^{2}$, Xianrong Liang ${ }^{2}$, Tianyun Dong ${ }^{2}$, Tao Liu ${ }^{2}$, Junhui Zhang ${ }^{2}$, \\ Jun Zou ${ }^{2}$, Huayong Yang ${ }^{2}$, and Chris Bowen ${ }^{2}$ \\ ${ }^{1}$ University of Bath \\ ${ }^{2}$ Affiliation not available
}

September 24, 2021

\begin{abstract}
Soft actuators and robotic devices have been increasingly applied to the field of rehabilitation and assistance, where safe human and machine interaction is of particular importance. Compared with their widely used rigid counterparts, soft actuators and robotic devices can provide a range of significant advantages; these include safe interaction, a range of complex motions, ease of fabrication and resilience to a variety of environments. In recent decades, significant effort has been invested in the development of soft rehabilitation and assistive devices for improving a range of medical treatments and quality of life. This review provides an overview of the current state-of-the-art in soft actuators and robotic devices for rehabilitation and assistance, in particular systems that achieve actuation by pneumatic and hydraulic fluid-power, electrical motors, chemical reactions and soft active materials such as dielectric elastomers, shape memory alloys, magnetoactive elastomers, liquid crystal elastomers and piezoelectric materials. Current research on soft rehabilitation and assistive devices is in its infancy, and new device designs and control strategies for improved performance and safe human-machine interaction are identified as particularly untapped areas of research. Finally, insights into future research directions are outlined.

Corresponding author(s) Email: mp351@bath. ac. uk, liutao@zju. edu. cn
\end{abstract}

\section{ToC Figure}




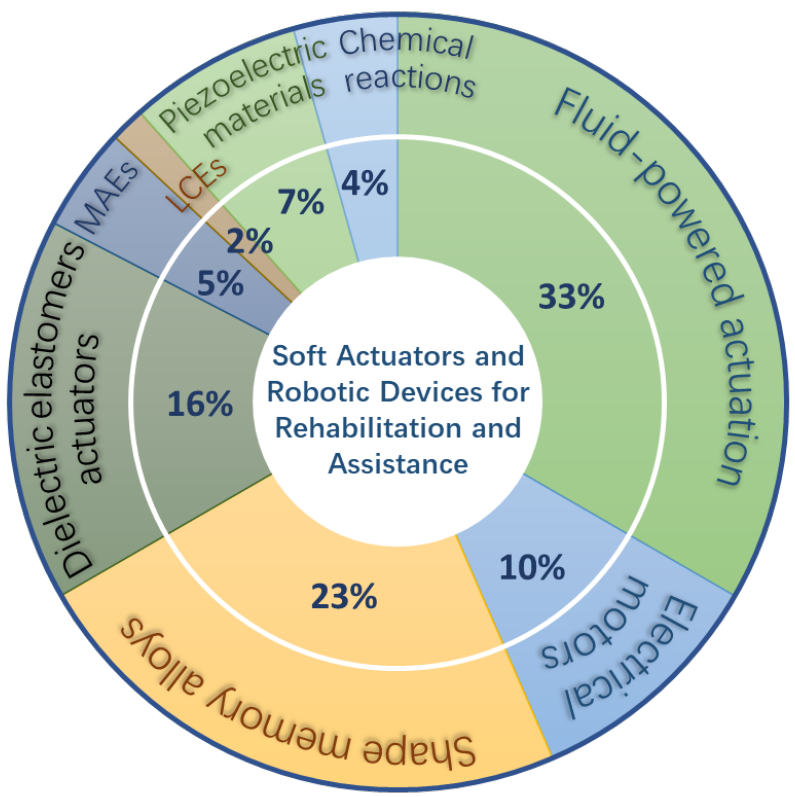

Figure 1: ToC Figure. Overview of the current state-of-the-art in soft actuators and robotic devices for rehabilitation and assistance, actuated using pneumatic and hydraulic fluid-power, electrical motors, chemical reactions, dielectric elastomers, shape memory alloys, magnetoactive elastomers (MAEs), liquid crystal elastomers (LCEs) and piezoelectric materials. The \% is the percentage of the number of the literatures with a certain actuation mechanism to the total number.

\section{Introduction}

Soft actuators and robotic devices can be considered as soft systems that aim to use highly compliant materials with elastic moduli that are comparable to soft biological materials and human tissues $(\mathrm{kPa}-$ $\mathrm{MPa}$ ). Such systems are highly compliant and able to perform a range of natural and flexible movements and are generally more adaptable and robust due to their high degrees of freedom (DoF) and flexible bodies. Soft materials employed in soft actuators and devices are often fabricated using low-temperature processes, thereby providing ease of processing and low-cost fabrication and their viscoelastic properties are able to dissipate energy from impact and damping oscillations to smooth out discontinuous motions and forces. These unique soft features provide advantages that have attracted increasing interest in the development of soft robotic devices for rehabilitation and assistance where safety during human-machine interaction is of primary importance.

While soft, cost-efficient, safe, and easy-to-use rehabilitation and assistive devices are highly desirable, the current state-of-the-art rehabilitation and assistive devices rely on the combination and control of rigid actuators, links, and joints to realise a range of motions. Despite the precise nature of rigid actuators, the range of available motion can be limited in terms of their DoF. This results in rigid systems being less efficient in terms of being able to adapt to a variety of operating conditions for different users. For example, a combination of a variety of electric motors may be needed to achieve a twisting motion. In contrast, a single well-designed soft actuator can readily realise both linear and twisting motions, providing the advantages of adaptability, a lightweight nature, ease-of-manufacture and cost-efficiency. Soft rehabilitation and assistive devices can therefore perform a complex range of motions by exploiting the potentially infinite DoF provided by soft actuators. However, for soft actuators and robotic devices to achieve their potential in rehabilitation and assistance, the underpinning technologies of sensing, actuation, control, and power supply must be fully 
integrated and operate cooperatively.

While there are a number of excellent reviews on soft robots [1-6], soft electronic-skins [7-9], flexible wearable devices [10-12], rehabilitation and assistive exoskeletons [13-16], soft systems in health care [17-20] and soft textile Exosuits [21], there is currently no review that has a focus on soft actuators and robotic devices specifically developed for soft rehabilitation and assistance. The aim of this review is to investigate the current state-of-the-art technologies in this area to demonstrate the nexus between materials, mechanisms, actuation, and applications. The range of available actuation mechanisms are reviewed and discussed; these include pneumatic and hydraulic fluid-powered actuation, electrical motor actuation, chemical reactions and soft active material-based actuation which includes dielectric elastomers, shape memory alloys, magnetoactive elastomers, liquid crystalline elastomers, and piezoelectric materials. Figure 1 summarises these areas and their contribution, in percent, to the existing academic literature. Fluid-powered actuation is one of the most popular methods for developing soft rehabilitation and assistive devices. Approximately $33 \%$ of the work in the field is based on this actuation technology as a result of its high power density, reliability, and controllability. The applications are widely spread and range from upper limb assistance (fingers, hands, arms) devices to lower limb rehabilitation (legs, knees, ankles, feet) devices. Magnetoactive elastomers (MAEs), liquid crystal elastomers (LCEs) and chemical reactions represent relatively new areas of research and, together, they represent approximately $11 \%$ of the work in the field, and the devices are mainly at the concept stage. The advantages, current limitations, and directions for future development of soft actuators and robotic devices for rehabilitation and assistance will be outlined in the final section of this review.

\section{Rehabilitation and assistance enabled by fluid-powered actuation}

Fluid power actuation systems, which use fluids under pressure to generate, control, and transmit power, are classified into two categories: pneumatic fluid power and hydraulic fluid power. Pneumatic systems use gas as a medium for power transmission, while hydraulic systems normally use a liquid, such as mineral oil or water. Such systems are able to produce high power (in the order of $\mathrm{kW}$ ) and high forces in small volumes, compared with electrically driven systems. For example, high fluid power of about $40 \mathrm{~kW}$ can be generated using a typical hydraulic gear pump operating at a pressure of 250 bar and a flow rate of $100 \mathrm{~L} / \mathrm{min}$, with a pump efficiency of $95 \%$. The rehabilitation and assistive devices enabled by pneumatic and hydraulic fluidpowered are overviewed in this section. Table 1 summarizes the details of the devices, including actuation mechanism, functionalities, key parameters, control strategies, advantages, and challenges.

\section{Pneumatic fluid-powered actuation}

Pneumatic fluid-powered actuators and robots have the advantages of flexibility, lightweight nature, safe interaction, ease of installation, and are comfortable to wear compared with their rigid counterparts. Pneumatic systems are usually made from soft materials such as polymers and fabrics, which can provide sufficient compliance, high force/volume ratio and a high degree of biocompatibility. A well-known example is the McKibben artificial muscle, which was invented by Richard H Gaylord [22] in 1958 and popularised by Joseph L McKibben at the beginning of 1960s. It has been used as a pneumatic actuator for orthotic systems [23-25] since the 1960s. However, the limited force $(100 \mathrm{~N}-300 \mathrm{~N})$ generated by the McKibben artificial muscle and the requirement of a compressed air tank limits its application to finger prehension orthosis. However, different designs in terms of muscle length, diameter, weaving angle and weaving fabric can significantly affect the static force of the McKibben muscle. With the development of advanced technologies in materials and actuation, the McKibben muscle was further developed and introduced into robotic devices to mimic the compliance of natural skeletal muscle by Bridgestone and other industrial companies in 1980s and 1990s [26]. Recently, with the growing need for safe interaction and flexibility, pneumatic fluid-powered artificial muscles and robots have been widely used for rehabilitation and assistive devices [13][16]. In this section, rehabilitation and assistive devices enabled by pneumatic fluid-powered actuators and robots that have been developed in the last ten years for use on both the upper and lower body are reviewed and discussed. 
Soft assistive devices, such as ankle-foot orthosis, have been developed for gait assistance and rehabilitation. Shorter et al. [27] developed a novel untethered ankle-foot orthosis (PPAFO) based on a pneumatic fluidpowered rotary actuator to provide untethered gait assistance for ankle-foot rehabilitation. A portable pneumatic power source, a rotary actuator, embedded electronics and sensors were integrated to construct an untethered device, as shown in Figure 2a. The total weight of the PPAFO was $3.1 \mathrm{~kg}$, which was distributed on a belt. The orthosis was able to provide plantar flexor and dorsiflexor torque assistance using a bidirectional pneumatic rotary actuator. The system positional response speed of the orthosis was $600^{\circ} / \mathrm{s}$ for a $0.98 \mathrm{~kg}$ load and $900^{\circ} / \mathrm{s}$ with no load, using a driven pressure of $0.62 \mathrm{MPa}$. The untethered ankle-foot orthosis demonstrates that the use of pneumatic fluid-powered actuators provides the capability to actuate a joint, with the advantages of a high force/weight and force/volume ratio. The results from non-disabled walking trials showed that a peak assistive torque of $9.2 \mathrm{Nm}$ can be achieved at an operating pressure of 0.62 $\mathrm{MPa}$, which validates that the PPAFO can provide sufficient plantar flexor assistance for gait modification.

Park et al. developed an active soft ankle-foot orthotics that was powered by pneumatic artificial muscles for gait assistance [28]. The device was fabricated with flexible and soft materials that mimic the muscletendon-ligament structure. The prototype weighed $950 \mathrm{~g}$ and was able to provide $12^{\circ}$ dorsiflexion from a resting position of an ankle joint and a $20^{\circ}$ dorsiflexion from plantarflexion. The active orthotics include three anterior muscles for assisting dorsiflexion in alleviating the drop-foot condition and can be expanded to include posterior muscles for actively assisting plantarflexion in a complete gait cycle. Thalman et al. [29] designed a soft one-size-fits-all ankle-foot orthosis (SR-AFO) using pneumatic soft fabric actuators to assist in inversion-eversion (IE) ankle support and gait rehabilitation, as shown in Figure 2b. The Exosuit was constructed from a custom neoprene fabric sleeve, which could be worn as a boot and adapted to the foot size of most adults. The soft fabric actuators were designed to operate vertically on both sides of the ankle, starting at the base of the talus and across the medial and lateral malleolus of the ankle. These actuators were deliberately used to mimic the placement of conventional ankle braces to prevent medial and lateral instability and provide IE ankle support. More importantly, the fabric actuators can be pneumatically controlled to increase IE ankle stiffness while not affecting ankle dorsiflexion-plantarflexion (DP) stiffness. The ability to actively vary the device stiffness provides an effective approach to prevent ankle buckling in the IE direction, while providing a comfortable, dynamic solution and behaving as a garment when inactive. Testing on six healthy subjects showed that the SR-AFO device could change eversion stiffness from approximately 20 to $70 \mathrm{Nm} / \mathrm{rad}$ at $30 \mathrm{kPa}$, while minimising the change in dorsiflexion stiffness. The variable stiffness of the soft pneumatic actuators makes the ankle-foot orthosis highly versatile and functional in multiple directions, as compared to conventional rigid motors. In addition, with an in-depth understanding of the kinematic and dynamic responses of the lower limb during walking, the controllability and portability of the SR-AFO can be further enhanced.

The development of soft wearable Exosuits is another area that is attracting increasing interest. A soft, ultralightweight lower-extremity robotic Exosuit for augmenting normal muscle function by $50 \%$ was developed by Wehner et al. [30]. The Exosuit was produced using a virtual anchor technique that connects McKibben pneumatic actuators through a soft network based on an inextensible webbing with triangulated attachment points. As shown in Figure 2c, pneumatic actuators are used to generate joint torques in the sagittal plane at the ankle, knee, and hip. This was achieved by connecting pneumatic muscles to the virtual anchor points (red points in Figure 2c) that were constrained using the soft inextensible webbing. The reaction force from the pneumatic muscles can be redirected to the connecting anchors. The pneumatic muscles used for the Exosuit were able to achieve a $90 \%$ of $\max$ force $(235 \mathrm{~N})$ in $0.316 \mathrm{~s}$ during inflation and a decrease from a maximum force to $10 \%$ of its maximum force in $0.098 \mathrm{~s}$ during deflation. The results demonstrated that the Exosuit had little effect on walking kinematics and the metabolic power $(386.7 \mathrm{~W})$, which is used to estimate the energy demands of acceleration and deceleration events of activities, which was almost identical to the average power $(381.8 \mathrm{~W})$ without the suit. The soft Exosuit is ultra-lightweight with a total weight of only $9121 \mathrm{~g}$, which has significantly reduced mechanical impedance and inertia compared with traditional rigid exoskeletons. Another lightweight wearable augmented walking suit (AWS) was developed by Thakur et al. [31], using pneumatic gel muscles (PGMs) that were able to generate assistive forces of $30 \mathrm{~N}$ at 60 
$\mathrm{kPa}$ and $44 \mathrm{~N}$ at $100 \mathrm{kPa}$ at an operating pressure range from 50 to $300 \mathrm{kPa}$. The walking suit provides both waist and knee support by attaching the PGM along the rectus femoris muscle. Force sensitive resistor sensors were arranged in the shoe to detect forces during the walking and thereby provide feedback to the assistive suit. A proportional feedback controller was used to switch on the PGMs to provide an assistive force during the swing phase of the human gait cycle, which was identified by the force-sensitive resistor (FSR) sensors placed in the shoes. This form of an augmented walking suit is untethered, portable, and lightweight with a total weight of $1.2 \mathrm{~kg}$. Experiments on subjects demonstrated that the AWS could achieve a significant reduction in the activity of most muscles, with a maximum of $44 \%$ reduction in the maximum voluntary contraction (MVC) of rectus femoris muscle at $60 \mathrm{kPa}$, and a maximum of $27.6 \%$ reduction in MVC of biceps femoris muscle at $100 \mathrm{kPa}$ while walking. The AWS is lightweight, portable and easy to use. To improve the controllability of the suit, inertial measurement units (IMUs) can be used to achieve improved motion detection for feedback control during a walking stance. It was noted that the AWS could be more user-friendly and comfortable by integrating the controller, battery, and air tank into the waist support belt.

Soft exosuits are also being developed for patient rehabilitation and disease prevention. For example, Deep Vein Thrombosis (DVT) is a severe medical condition that can affect patients who are bed-ridden for a long period after a stroke. Blood clots in the deep veins of the lower extremity can significantly affect and deteriorate normal blood flow. The current state-of-the-art methodologies to prevent DVT can be categorised as pharmacological prophylaxis and mechanical prophylaxis. While the pharmacological prophylaxis uses anticoagulant drugs to prevent blood clotting, mechanical prophylaxis systems are normally developed to promote venous blood flow and resolve the venous stasis. However, commercial mechanical prophylaxis devices have led to side effects such as skin necrosis, blisters, and injury due to rigid patient-machine interactions. A soft wearable suit has therefore been considered as a new approach to improve the patient experience and tackle challenges associated with DVT. Low et al. [32] developed an Exosock for DVT, primarily as an alternative to mechanical prophylaxis. The Exosock was driven by soft double-extension pneumatic actuators which can be attached to the lower extremity of the body, as shown in Figure 2d. The soft Exosock consists of five different modules including a sock, knee sleeve, soft double-extension actuators placed within a fabric, joint angle sensor and a programmable pump-valve controller. When the actuator is inflated, it extends and guides the foot in a plantarflexion motion; when the actuator is deflated, it retracts to its original length in which the resultant tension assists in dorsiflexion motion of the ankle. An inertial measurement unit (IMU) was attached to the metatarsal region of the foot to measure the ankle joint angle in real-time and provide feedback to the controller. The soft actuator was able to generate a peak force of $33.2 \pm 0.3 \mathrm{~N}$ at $100 \%$ strain. Pilot results on a healthy subject showed that the Exosock was able to achieve an average of $16.4 \pm 1.3^{\circ}$ of passive ankle dorsiflexion consistently with low deviation, which provides a promising solution for DVT prevention. The real-time feedback and the wireless control capabilities of the Exosock can allow therapists or doctors to monitor ankle exercises and control the Exosock remotely; however the overall efficacy of the device for DVT prevention needs to be quantified with the use of clinical trials.

Total knee arthroplasty (TKA) is a surgical process to restore the knee joint function by replacing a damaged, worn, or diseased knee with an artificial joint. After a TKA procedure, patients often have a reduced range of motion and low quadricep strength; there is therefore a need for an assistive device. However, conventional metal-based exoskeletons have size and weight limitations. As a solution, a lightweight pneumatic exoskeleton for TKA patients was developed by Ezzibdeh et al. [33] that used a fabric pneumatic actuator as the bending joint. The exoskeleton consisted of a plastic structure body with on-board electronics, a power pack in the waist belt, a knee joint enabled by the fabric pneumatic actuator, and four Velcro straps to secure the device in place, as seen in Figure 2e. The device had a built-in force-feedback controller and a non-linear spring to assist in detecting the intent of the patient to move and provide continuous assistance. The positive results showed that the pneumatic exoskeletons are promising in assisting patients after TKA procedures.

Baiden and Ivlev [34] proposed a concept of an active human-robot-interaction (HRI) control for orthoses using soft pneumatic actuators. A 2-DoFs exoskeleton robot was applied to implement the concept and analyse HRI control strategies. The robot was comprised of two lower extremities orthoses equipped with 
soft actuators that were directly attached to human knee and ankle joints, as shown in Figure 2f. To realise rotary motion and allow the device to mimic human kinematics, a soft pneumatic actuator with pleated rotary elastic chambers (REC) was developed and used to create the orthoses [35]. A knee joint that combined a series of two REC-actuators were able to achieve a full range of motion from $0^{\circ}$ to $90^{\circ}$, while at the ankle joint a single REC-actuator was able to rotate from $0^{\circ}$ to $45^{\circ}$. A master-slave with a compliant position control strategy was developed for HRI, in which a reference movement was achieved from the healthy leg as the master, and the desired control signals were generated and transferred to the impaired leg to achieve precise movement. This approach can be highly suitable for hemiplegia patients who are paralysed on one side of their body but are able to readily move their limbs on the unaffected body part and balance using a support frame. The HRI concept was proven using human sit-to-stand and stand-to-sit examples. The controllable stiffness of the REC actuators was also exploited to achieve a spring-like behaviour, with an independent stiffness-position or stiffness-torque control to further enhance the HRI concept [36]. A linear relationship between the torque and driven pressure was assumed for the REC systems, independent stiffnessposition and stiffness-torque control can be realised using a position and torque look-up table determined from experimental calibration, which effectively includes any non-linearities of the system. The desired torque and stiffness need to be accurately predicted to maintain an appropriate pressure within the supply pressure range and the torque-angle hysteresis will need to be compensated.

Fang et al. designed a foldable pneumatic bending actuator (FPBA), which were fabricated using thermoplastic polyurethane fabric materials and developed for an FPBA-based knee Exosuit [37]. FPBAs were formed in a bellow structure and could be folded into fan shapes to achieve different folding angles from 0 to $360^{\circ}$. With an increase in pressure from 0 to $40 \mathrm{kPa}$, the folding angle was varied from $30^{\circ}$ to $180^{\circ}$, and the output torque was up to $25.74 \mathrm{Nm}$. The Exosuit consisted of an onboard electronic and sensing system, an off-board pneumatic and control system, and a knee sleeve that attached the FPBA behind the user's knee. Five healthy subjects were recruited to test the knee Exosuit. The results showed that FPBAs were able to generate bending motions with a large range of motion that could achieve human-scale torque levels at a low input pressure (up to $40 \mathrm{kPa}$ ), which can effectively assist in knee movement. The FPBA can bend at any angle without airflow restrictions with the advantages of low cost, ease of manufacturing, mechanical flexibility, and comfortability. However, the FPBA is currently tethered and not portable since it employs a miniature pump; however, the design of a compact power supply could enable an untethered FPBA to be produced. Furthermore, human intention recognition, such as biomechanical information recognition and surface electromyography recognition, can be used to realise human-robot-interaction control, which can improve the controllability of the FPBA.

Pneumatic fluid-powered actuation is widely used in lower body rehabilitation and assistance. The McKibben pneumatic muscle is one of the popular choices for orthotic devices and lower extremity Exosuits [28, 30]. They are lightweight, cost-efficient and have low mechanical impedance and inertia. They can provide a reasonably large force (e.g. $370 \mathrm{~N}$ [30]) with a small pressure (e.g. 5 bar [30]) and exhibit a rapid response time $(0.01 \mathrm{~s}-1 \mathrm{~s}[30])$. In addition, they are normally tethered and need the auxiliary power device to operate the system.

Pneumatic rotary and foldable actuators are also welcomed by the community [27, 33-38] due to their flexibility of motion. They can operate with a large range of bending degrees (e.g. $0^{\circ}$ to $360^{\circ}$ for a foldable actuator [37]), which are suitable for a wide range of rehabilitation and assistance applications, including simultaneous dorsiflexor assistance and plantar flexor assistance, TKA and post-surgery rehabilitation, and knee and ankle movement assistance.

Fabric-based pneumatic Exosuits have shown their advantages of varying stiffness, portability, and comfortability and ultra-lightweight [29]. Compared with McKibben pneumatic muscle-based devices, they generally operate at lower pressures (e.g. 0.5 bar [29]), in which lower forces are expected. These can be highly suitable for small assistance tasks, but they may not be sufficient for medical rehabilitation programmes due to limited force. However, the successful concept indicates a promising research direction of the development of soft wearable suit for our daily use. These assistive devices can significantly reduce the required muscle 
(a)

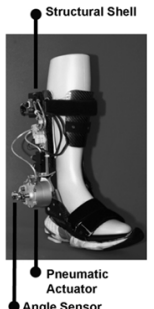

(c)

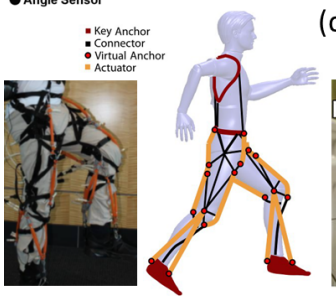

Polenoid
Valves

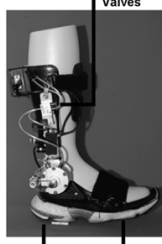

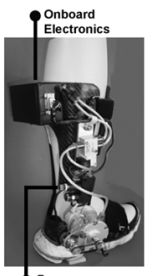

(d)

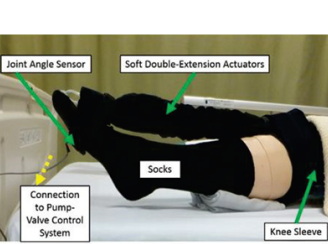

(b)
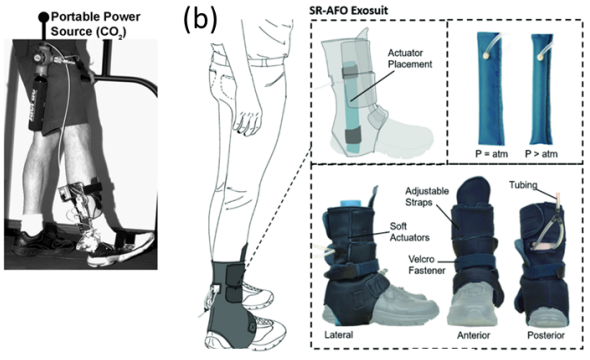

(e) (f)

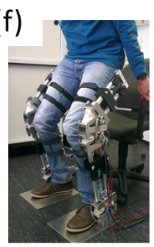

Figure 2: Pneumatic fluid-powered rehabilitation and assistive devices for lower body rehabilitation and assistance (a) untethered ankle-foot orthosis (PPAFO) driven by a pneumatic rotary actuator [27] (b) soft robotic ankle-foot orthosis SR-AFO exosuit driven by pneumatic soft fabric actuators [29] (c) soft lowerextremity robotic exosuit for augmenting normal muscle function [30] (d) soft ankle-foot exosock for deep vein thrombosis [32] (e) soft pneumatic exoskeleton for after total knee arthroplasty (TKA) [33] (f) lightweight exoskeleton using a fabric pneumatic actuator as the bending joint [34].

effort and empower our capability in daily activities.

Real-time feedforward and feedback control systems (e.g. PID control [28], force feedback control [31,33], human-robot interaction control [34, 36], patient-centred control [35]) have been developed for use as lower body rehabilitation and assistive devices. However, control accuracy and reliability continue to be key challenges, which are caused by the inherent compliance of the soft actuators. In addition, uncertain and inaccurate gait feedback can lead to large perturbation of the control systems, which can lead to wearer discomfort, or even injury. To address this challenge, advanced control algorithms and optimisation strategies can be employed.

New soft pneumatic fluid-powered actuators have also been developed and applied for upper extremity rehabilitation and assistance. Wilkening et al. designed a soft elbow trainer using soft pneumatic actuators as bending joints, as shown in Figure 3a [38]. The elbow trainer consisted of a bending joint, a passive rotation joint, and a passive translation joint to realize motion in both extension and flexion directions with an implicit self-alignment to the polycentric movement of a human joint. The bending joint was constructed using three pneumatic skewed rotary elastic chambers (sREC) actuators [35] connected in series with two antagonistic rotary elastic chambers. The bellows of the actuators were pneumatically connected, which were able to achieve a bidirectional rotary motion with a range of $120^{\circ}$. An artificial neural network (ANN) was used to estimate the trainer position using sensing signals from four flexure sensors. The estimated position from the ANN was experimentally examined and compared with the measured results from the inertial measurements units (IMUs), showing an average of the absolute signal difference of $1.25^{\circ}$ with a standard deviation of only $0.85^{\circ}$. The adaptive assistance combined with the self-alignment capability can significantly reduce the device adjustment time. The adaptive position feedforward and feedback control enables the elbow trainer to provide adaptive assistance and human-robots interaction without using the torque and stiffness information.

For hand rehabilitation applications, Li et al. [39] developed an assistive glove that was combined with a pneumatic actuator fabricated using a latex material for post-stroke patients. The device consisted of a power pack, control electronics, a curvature sensor, and a soft "ball" shape pneumatic actuator. The actuator was 
embedded into the surface of the glove to control the bending angle of a finger, which could be measured by embedded curvature sensors. Sensors were calibrated based on a three-layer back-propagation neural network which achieved an accuracy rate of $91 \%$. A closed-loop proportional controller was developed, and experimental results showed the device was able to quickly track the desired position in approximately $1 \mathrm{~s}$. The device can only move all fingers together and the nonlinearity and resulting hysteresis of the system needs to be considered and addressed in the design of the controller. To independently control fingers and assist with functional grasp pathologies, Yap et al. [40] developed a soft robotic glove that was driven by fibre-reinforced elastomer actuators (FEAs). The FEAs were mechanically programmed to generate the desired motion paths by tailoring their morphology. The actuation force of the FEAs was up to $13.6 \mathrm{~N}$ at an actuation pressure of $153 \mathrm{kPa}$ and each glove finger could be independently actuated by an individual FEA to realise a full range of hand motions. Radio-frequency identification (RFID) tags were combined with surface electromyogram (sEMG) electrodes in a brace to measure the movement of the forearm muscles and an EMG-RFID control strategy was developed where the RFID tags were used as non-physical switches for tasks such as palmar grasp and pincer grasp while the sEMG signals from the forearm muscles were used to monitor the states of the hand including activate, hold and release. The results showed that the glove could achieve palmar and pincer grasps corresponding to a Kapandji Score of 9. A Kapandji Score (score 1 to 10) was used to assess the movement of the opposition of the thumb with the other fingers. The EMG-RFID controller enabled the glove to detect user intention with the minimum number of sEMG electrodes on the device. To simplify the sensing system, a forearm band with three force-sensitive resistors was developed to capture the force myography (FMG) signals from three muscle locations [41]. An artificial neural network (ANN) was used to classify four hand configurations, including finger extension, finger flexion, wrist extension and wrist flexion from the measured FMG data. The results showed that the average real-time testing accuracy could reach $94.04 \%$ with a standard deviation of $2.06 \%$, which is feasible and promising for successful user-interaction control for hand rehabilitation. The team also developed a bidirectional pneumatically driven glove that can provide both active flection and extension [42]. The glove is fully fabric-based and includes both the flexion and extension actuator fabricated by using ultrasonic welding layers of thermoplastic polyurethane (TPU) coated fabric. The flexion actuator can achieve a bending radius of $0.069 \pm 0.003$ at $30 \mathrm{kPa}$ and a block tip force of $14.3 \mathrm{~N}$ at $70 \mathrm{kPa}$ while the extension actuator can provide an extension torque of $0.3 \mathrm{Nm}$ at $70^{\circ}$. Tests on five healthy participants showed that the glove was sufficient for $90 \%$ of daily function activities. Compared with the fabric-regulated elastomeric gloves, the fully fabric-based gloves are much lighter and can achieve a smaller bending radius, a larger range of motion, and higher forces output at lower pressures. The use of the extension actuators can effectively mitigate the slow dynamic responses due to pneumatic valve discharging and achieve bidirectional motions, although fatigue tests on the fabric-based actuators and the evaluation of efficacy on impaired patients need to be conducted. For thumb rehabilitation, Shiota et al. developed a device by integrating the FEAs into a 5-digit assist system which is assembled onto a forearm socket [43]. The FEAs were able to bend over $170^{\circ}$ in $0.5 \mathrm{~s}$ and return to a resting state within $0.4 \mathrm{~s}$. The average maximum torque of the FEA was $36.1 \mathrm{cNm}$. An enhanced Kapandji test was conducted, and the results showed that the gloves can reach the majority of positions that are commonly used on a daily basis.

Powered wearable assistive devices are used for helping carers during repetitive and heavy work. Lower back pain (LBP) is a common symptom, which is related to repetitive and heavy lifting and bending. Soft wearable power assistance devices manufactured from soft pneumatic actuators provide a promising solution to meet this important societal need. A powered assistive device that used two types of pneumatic actuators for the tasks of lifting and holding to prevent LBP was developed by Cho et al. [44]. The device employed an elongation-type pneumatic actuator and layer-type pneumatic actuator to provide an assistive force for the lower back, as shown in Figure 3b. The elongation-type actuator was constructed using a rubber tube with a woven bellows sleeve cover with two ties at each end. The actuator was able to extend from an initial length of $320 \mathrm{~mm}$ to $490 \mathrm{~mm}$ at a driven pressure of $500 \mathrm{kPa}$. The layer-type actuator consisted of two thermoplastic polyurethane (TPU) balloons inside nylon pockets, which was able to extend its height from an original length of $2 \mathrm{~mm}$ to $50 \mathrm{~mm}$ at a driven pressure of $250 \mathrm{kPa}$. The expansion force of the layer-type actuator could reach up to $450 \mathrm{~N}$ at a pressure of $60 \mathrm{kPa}$. A feedback PID control system was developed using the actuator pressure and body acceleration and the acceleration was measured when the human body 
was inclined. The results showed that the desired pressures could be achieved, and a maximum pressure of $500 \mathrm{kPa}$ can be achieved with a rise time of $2.5 \mathrm{~s}$. The deflation time from the pressure of $100 \mathrm{kPa}$ to 0 was $1.7 \mathrm{~s}$. The device was used by three subjects, and the results showed it was able to effectively reduce the maximum muscle activity by $33.1 \%$ and the muscle activity time by $2.16 \mathrm{~s}$. The device is lightweight, user friendly and can be worn on the body with everyday clothing, demonstrating the benefits of a non-rigid and soft skeleton frame structure.

Another soft body suit was reported by Abe et al. that was based on combining two novel concepts (i) a muscle textile and (ii) shifts in the balancing posture of the body [45], as shown in Figure 3c. The active muscle textile was fabricated by sewing multiple soft, thin pneumatic muscles into a flexible fabric, which provided high power density, high force, good flexibility and a lightweight nature. The suit consisted of 11 muscle textiles and was divided into two parts to support shoulder flexion and horizontal flexion, elbow flexion and extension, respectively. The suit weighed $2 \mathrm{~kg}$ and its high force capability was able to support and disperse the operating stress at the contact points between the human body and support unit, which effectively reduced the force on the human body and improved comfort. The flexibility and lightweight nature of the textile actuators enabled complex motions, such as twisting. To evaluate its performance, the device was used by a subject and the reduction of the body burden force was evaluated by measuring the integrated electromyogram (iEMG) signals of the muscles. The results showed a burden reduction of $33 \%$ in the biceps brachialis muscle and a suppression of $5 \%$ in the body sway. The muscle textile was recently further improved using a novel ' 18 weave' structure muscle [46], where Figure $3 \mathrm{~d}$ shows the ' 18 weave' structure that is weaved using thin McKibben muscles as warps and wefts. The ' 18 weave' structure muscle was able to retract by $26.5 \%$ of its original length. It was observed that during use of the iEMG of posterior deltoid decreased by $23 \%$, and the myoelectric potentials of the anterior deltoid decreased by $40 \%$.

Ang et al. presented a 3D printed Soft Robotic Wrist Sleeve (SWS) with 2-DoFs in the flexion-extension and radial-ulnar direction for wrist rehabilitation [47]. Two fold-based soft pneumatic actuators were attached to the fabric sleeve to realise 2-DoFs motion, as shown in Figure 3e. The SWS was able to achieve a 71.1\% range of motion on healthy wrists and could provide sufficient torque and bending curvature at an operating pressure of $200 \mathrm{kPa}$. The SWS is easy to fabricate using 3D printing technology and can assist with wrist in both flexion-extension and radial-ulnar directions.

Soft pneumatic fluid-powered actuators have also been developed and applied for upper extremity rehabilitation and assistance. They are used for assistance and rehabilitation of fingers [43], hands [39-42], wrists [47], and elbows [38], and support of upper limbs [45,46] and the back [44]. They are lightweight and costefficient, but more complex to control for a variety of motions. For example, controlling the devices for different finger movements synchronously and accurately is challenging. Real-time feedforward and feedback control systems (e.g. PID control [39], adaptive assistive control [38], integrated electromyography (EMG) -radio-frequency identification (RFID) control [40]) have been developed and used for the devices to achieve desired performance. To increase the comfortability and wearability, fabric-based pneumatic actuators are popular, and they can provide a reasonable force range for hand movement (e.g. $14.3 \mathrm{~N}$ at $70 \mathrm{kPa}$ [42]) and perform a wide range of motion. In addition, McKibben pneumatic muscle-based devices are also developed for higher force (e.g. $450 \mathrm{~N}$ at $60 \mathrm{kPa}$ [44]) for support purposes, such as lower back and upper limb support. Advanced manufacturing technologies, such as 3D-printed technologies, has also been used in the field. The soft robotic wrist sleeve (SWS) is a promising example which provides flexible foldable actuators, which has significant potential for the development of cost-efficient and easy-to-manufacturing assistive devices. It will enhance our capability for 'making it ourselves' assistive devices at home for daily activities.

\section{Hydraulic fluid-powered actuation}

Hydraulic fluid-powered systems provide a high-power density by pressurising fluids of high viscosity and low compressibility in their actuators; the power density is particularly high when compared to pneumatic fluid-power systems which often use air as the fluid medium. Hydraulic fluid-powered actuation therefore combines the advantages of high-power density (e.g. $180 \mathrm{~kW} / \mathrm{m} 3$ ), fast dynamic response (within a few 

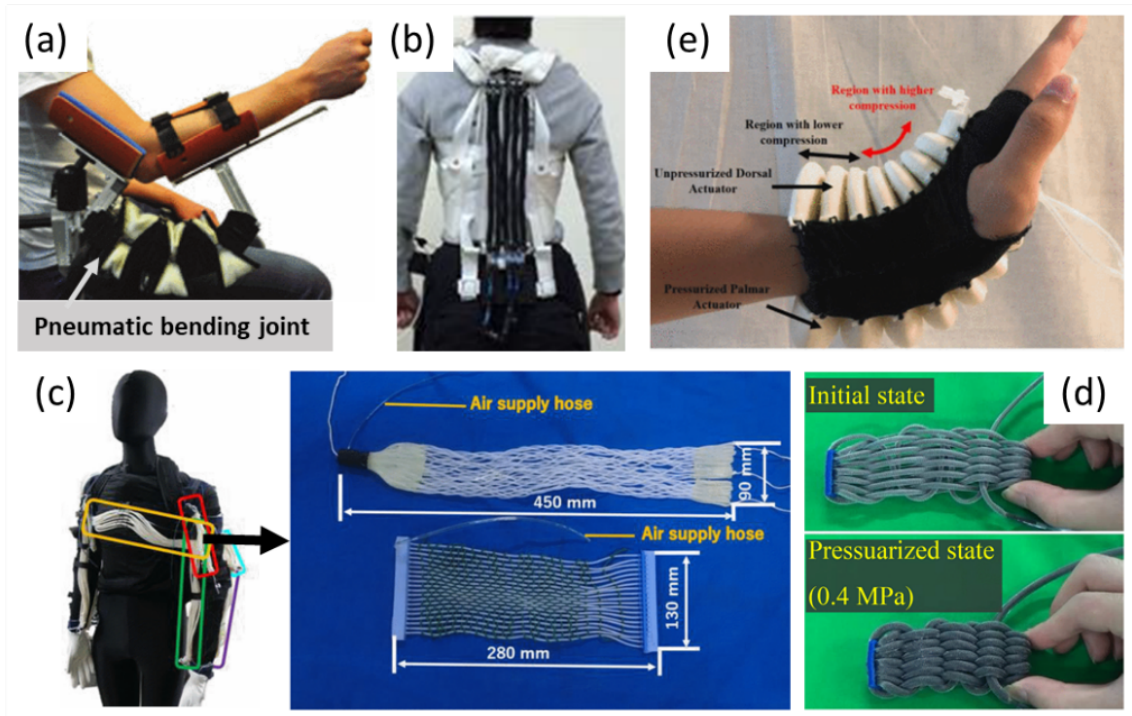

Figure 3: Pneumatic fluid-powered rehabilitation and assistive devices for upper body rehabilitation and assistance. (a) soft elbow trainer using soft pneumatic bending joints [38] (b) soft wearable assistive device for low back support (LBP) [44] (c) soft muscle textile-based power support suit for upper limbs [45] and (d) new '18 weave' fabrication approach [46].

millisecond), and good controllability. This actuation mechanism has been used for soft rehabilitation and assistive devices. Polygerinos et al. [48] developed a soft robotic glove using fluid-driven fibre-reinforced elastomer actuators to augment hand rehabilitation for individuals with functional grasp pathologies, as shown in Figure 4. The soft actuators were mechanically programmed using a specific arrangement of fibres and limit layers to match the range of motion (ROM) of human hands. To operate the soft robotic glove, a closed-loop control system was designed to regulate the actuation pressure and a sliding mode control was used to control the soft actuator precisely. The actuator was able to respond to a step response within 0.2 $\mathrm{s}(65 \%)$ and reach a steady-state after $2.2 \mathrm{~s}(95 \%)$. A qualitative test showed that the glove could fulfil the range of activities need for daily life where the glove was used by a healthy individual. The results showed that the glove could generate sufficient force to perform grasping a $500 \mathrm{~g}$ tin can without biological muscle effort. The glove was also evaluated by a participant with muscular dystrophy and preliminary testing showed that the participant could effectively grasp, hold, and release a wooden block using a glove with sEMG control. The actuator was also customised based on the user's biomechanics and evaluated by a motion capture system, showing an almost equivalent ROM to the user's biomechanics [49]. Preliminary evaluation of the glove on a patient with reduced hand function was also conducted, and the result showed that the patient can perform tasks faster with a greater degree of functional grasping in a standardized Box-and-Block test with the assistance of the sEMG controlled glove. 


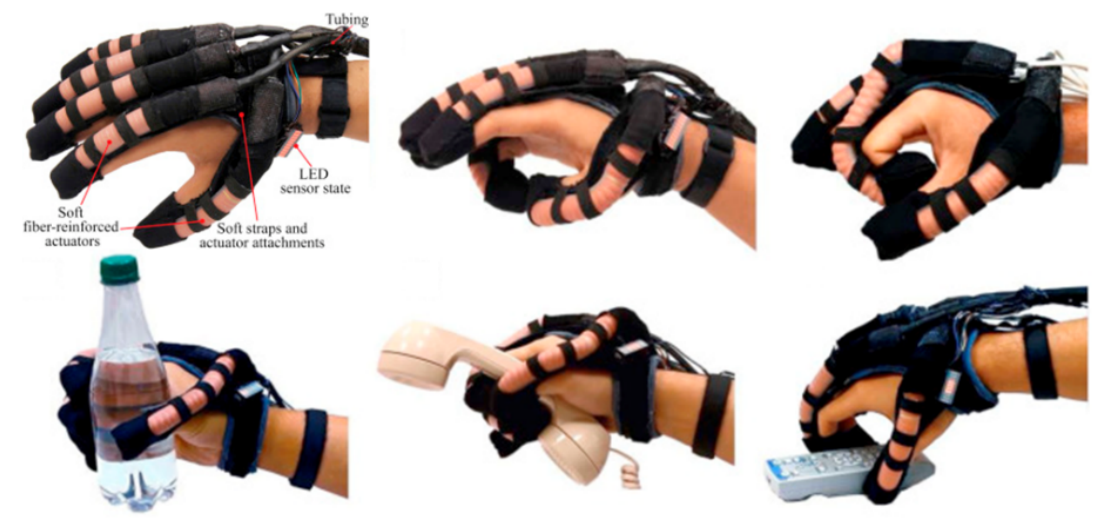

Figure 4: Hydraulic fluid-powered soft gloves for rehabilitation and assistance [48][49] 


\begin{tabular}{|c|c|c|c|c|c|c|c|}
\hline $\begin{array}{l}\text { Rehabili- } \\
\text { tation and } \\
\text { assistive } \\
\text { devices } \\
\text { Pneu- } \\
\text { matic } \\
\text { fluid- } \\
\text { powered } \\
\text { actuation }\end{array}$ & Actuators & $\begin{array}{c}\text { Functionali- } \\
\text { ties }\end{array}$ & $\begin{array}{c}\text { Key } \\
\text { parameters }\end{array}$ & $\begin{array}{c}\text { Control } \\
\text { strategies }\end{array}$ & $\begin{array}{l}\text { Advan- } \\
\text { tages }\end{array}$ & $\begin{array}{l}\text { Chal- } \\
\text { lenges }\end{array}$ & Refs. \\
\hline $\begin{array}{c}\text { Portable } \\
\text { powered } \\
\text { ankle-foot } \\
\text { orthosis } \\
\text { (PPAFO) }\end{array}$ & $\begin{array}{l}\text { Pneumatic } \\
\text { rotary } \\
\text { actuator }\end{array}$ & $\begin{array}{l}\text { Untethered, } \\
\text { simultaneous } \\
\text { dorsiflexor } \\
\text { assistance } \\
\text { and plantar } \\
\text { flexor } \\
\text { assistance }\end{array}$ & $\begin{array}{c}\text { System } \\
\text { response } \\
\text { speed: } 600^{\circ} / \mathrm{s} \\
(0.98 \mathrm{~kg} \\
\text { load }), 900^{\circ} / \mathrm{s} \\
(\text { no load }) \text { at } \\
0.62 \mathrm{MPa} \\
\text { Assistive } \\
\text { torque: } 9.2 \\
\text { Nm at } 0.62 \\
\text { MPa } \\
\text { Weight: } 3.1 \\
\text { kg }\end{array}$ & $\begin{array}{c}\text { Magnitude } \\
\text { and timing } \\
\text { torque } \\
\text { control }\end{array}$ & $\begin{array}{l}\text { High } \\
\text { force/weigl } \\
\text { Flexibil- } \\
\quad \text { ity; }\end{array}$ & $\begin{array}{l}\text { Inaccu- } \\
\text { t,racy of } \\
\text { gait } \\
\text { events } \\
\text { detec- } \\
\text { tion }\end{array}$ & $\begin{array}{l}\text { Shorter } \\
\text { et } \\
a l . \\
{[27]}\end{array}$ \\
\hline $\begin{array}{c}\text { Bio- } \\
\text { inspired } \\
\text { active soft } \\
\text { orthotic } \\
\text { device }\end{array}$ & $\begin{array}{l}\text { McKibben } \\
\text { pneumatic } \\
\text { actuator }\end{array}$ & $\begin{array}{l}\text { Rehabilita- } \\
\text { tion for } \\
\text { ankle foot } \\
\text { pathologies }\end{array}$ & $\begin{array}{c}\text { Dorsiflexion: } \\
12^{\circ}-20^{\circ}\end{array}$ & $\begin{array}{c}\text { Feedforward } \\
\text { control } \\
\text { system, }\end{array}$ & $\begin{array}{l}\text { Lightweigh } \\
\text { low cost }\end{array}$ & $\begin{array}{l}\text {;Unteth- } \\
\text { ered } \\
\text { opera- } \\
\text { tion; } \\
\text { control- } \\
\text { ability }\end{array}$ & $\begin{array}{l}\text { Park } \\
\text { et } \\
\text { al. } \\
{[28]}\end{array}$ \\
\hline & & & $\begin{array}{l}\text { Weight: } 950 \\
\mathrm{~g}\end{array}$ & $\begin{array}{c}\text { feedback } \\
\text { proportional } \\
\text { control }\end{array}$ & & & \\
\hline $\begin{array}{c}\text { Soft } \\
\text { robotic } \\
\text { ankle-foot } \\
\text { orthosis } \\
\text { (SR- } \\
\text { AFO) } \\
\text { exosuit }\end{array}$ & $\begin{array}{l}\text { Fabric-based } \\
\text { pneumatic } \\
\text { actuator }\end{array}$ & $\begin{array}{c}\text { Inversion } \\
\text { and eversion } \\
\text { ankle } \\
\text { support }\end{array}$ & $\begin{array}{c}\text { Eversion } \\
\text { stiffness: } \\
20-70 \\
\mathrm{Nm} / \mathrm{rad} \text { at } \\
\text { ¡30 kPa, } 84.9 \\
\mathrm{Nm} / \mathrm{rad} \text { at } 50 \\
\mathrm{kPa}\end{array}$ & $\begin{array}{l}\text { Feed- } \\
\text { forward } \\
\text { control } \\
\text { system }\end{array}$ & $\begin{array}{l}\text { Varying } \\
\text { stiffness }\end{array}$ & $\begin{array}{l}\text { Control- } \\
\text { ability } \\
\text { and } \\
\text { portabil- } \\
\text { ity }\end{array}$ & $\begin{array}{l}\text { Thal- } \\
\text { man } \\
\text { et } \\
\text { al. } \\
{[29]}\end{array}$ \\
\hline $\begin{array}{l}\text { Lightweight, } \\
\text { soft lower } \\
\text { extremity } \\
\text { exosuit }\end{array}$ & $\begin{array}{l}\text { McKibben } \\
\text { pneumatic } \\
\text { actuator }\end{array}$ & $\begin{array}{l}\text { Augmenta- } \\
\text { tion of } \\
\text { normal } \\
\text { muscle } \\
\text { function for } \\
\text { gait } \\
\text { assistance }\end{array}$ & $\begin{array}{c}\text { Actuator } \\
\text { force: } 370 \mathrm{~N} \\
\text { at } 5 \text { bar, }\end{array}$ & $\begin{array}{c}\text { Feedforward } \\
\text { control } \\
\text { system }\end{array}$ & \multicolumn{2}{|c|}{$\begin{array}{l}\text { Lightweight, Design } \\
\text { opti- } \\
\text { mization } \\
\text { for } \\
\text { sufficient } \\
\text { assis- } \\
\text { tance } \\
\text { power }\end{array}$} & $\begin{array}{l}\text { Wehner } \\
\text { et } \\
\text { al. } \\
{[30]}\end{array}$ \\
\hline & & & $\begin{array}{c}\text { Response } \\
\text { time: achieve } \\
90 \% \text { of } \max \\
\text { force }(235 \mathrm{~N}) \\
\text { in } 0.32 \mathrm{~s}, \\
\text { drop to } 10 \% \\
\text { in } 0.1 \mathrm{~s} \text { at } 4 \\
\text { bar, } \\
\text { Weight: } 9121 \\
\mathrm{~g}\end{array}$ & & $\begin{array}{l}\text { low me- } \\
\text { chanical } \\
\text { impedance } \\
\text { and } \\
\text { inertia }\end{array}$ & & \\
\hline $\begin{array}{c}\text { Soft } \\
\text { wearable } \\
\text { aug- }\end{array}$ & $\begin{array}{l}\text { Pneumatic } \\
\text { gel muscle }\end{array}$ & $\begin{array}{l}\text { Reduction of } \\
\text { required } \\
\text { muscle effort }\end{array}$ & $\begin{array}{l}\text { Actuator } \\
\text { force: } 30-44 \\
\mathrm{~N} \text { at about }\end{array}$ & $\begin{array}{l}\text { Force- } \\
\text { feedback } \\
\text { based }\end{array}$ & $\begin{array}{l}\text { Light, } \\
\text { portable, } \\
\text { easy to }\end{array}$ & $\begin{array}{l}\text { Gait } \\
\text { cycle de- } \\
\text { tection }\end{array}$ & $\begin{array}{l}\text { Thakur } \\
\text { et } \\
\text { al. }\end{array}$ \\
\hline
\end{tabular}




\section{Rehabilitation and assistance enabled by electrical motors}

Over the last two decades, a number of soft robotic powered exosuits have been developed at the Harvard Biodesign Lab. These soft systems have demonstrated an ability to augment strength and reduce the level of loading experienced by the wearer. The exosuits use pneumatically actuated and electrical motor actuated systems. The pneumatic-driven exosuit is constructed using McKibben muscles which are attached to the exosuit using a virtual anchor technique (see Figure 2c) to assist hip, knee, and ankle movement [30]. The core device is lightweight and weighs $3.5 \mathrm{~kg}$ without peripherals.

An alternative actuation approach uses high-power electrical motors driving Bowden cables, which enables the development of untethered exosuits [50]. The exosuits used garment-like functional textile anchors that are worn around the waist and calf and Bowden cable-based mechanical power transmissions to generate assistive joint torques as a function of the paretic gait cycle, as shown in Figure 5. A force-based control system was developed for real-life exosuits, with wearable strain sensing elements for human walking and running motion measurements [51][52]. An investigation and evaluation of the first-generation multi-articular, portable, and fully autonomous soft exosuit was conducted by Asbeck et al [53]. The results showed that an exosuit can provide a net metabolic increase of $9.3 \%$. A breakthrough in the development of a soft robotic powered exosuit for post-stroke patients was presented by Awad et al [54]. The overall mass of the exosuit was approximately $0.9 \mathrm{~kg}$ and the light exosuits can effectively reduce interlimb propulsion asymmetry, increase ankle dorsiflexion, and reduce the energy required during walking. The exosuits were able to function synchronously with a wearer's paretic limb to facilitate an immediate $5.33 \pm 0.91^{\circ}$ increase in the paretic ankle's swing phase dorsiflexion and a $11 \pm 3 \%$ increase in the paretic limb's generation of forward propulsion $(\mathrm{P}<0.05)$. This resulted in a $20 \pm 4 \%$ reduction in forward propulsion interlimb asymmetry and a $10 \pm 3 \%$ reduction in the energy cost of walking, which is equivalent to a $32 \pm 9 \%$ reduction in the metabolic burden associated with poststroke walking. To optimise device performance, Ding et al. optimised the control of a soft exosuit developed for hip assistance during walking using a human-in-the-loop Bayesian optimisation method [55]. When wearing the optimised exosuit, the metabolic energy can be reduced by 17.4 $\pm 3.2 \%$, which showed a significant improvement of more than $60 \%$ on metabolic reduction compared with state-of-the-art hip assistive devices. The relationship between assistance magnitude from the exosuit, the metabolic cost during walking and the gait mechanics was investigated by Quinlivan et al. [56]. The results show that the net metabolic rate of walking decreased by $22.83 \pm 3.17 \%$ at a maximum exosuit assistance. Soft exosuits driven by electrical motors have great potential to assist our daily activities and increase walking efficiency, as well as demonstrating future potential for rehabilitation applications. Such systems are lightweight, textile-based, untethered and have integrated sensing elements and controllers, which are ideal for applications related to mobility assistance.
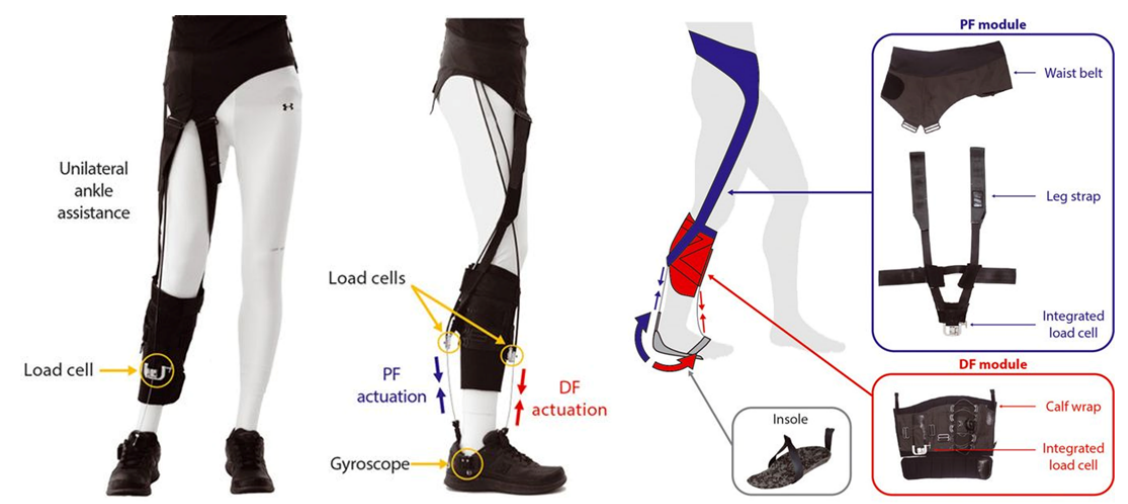

Figure 5: Garment-like soft exosuit for lower limbs assistance [54] 


\section{Rehabilitation and assistance enabled by soft active materials}

Soft active materials, including shape memory alloys, dielectric elastomers, magneto-active elastomers, liquid crystalline elastomers, and piezoelectric materials, have been used to develop soft rehabilitation and assistive devices. Table 2 summarises the details of these devices, including the materials employed, actuation mechanisms, key function and operating parameters, applications, their advantages, and the challenges faced with respect to future development.

\section{Shape memory alloys (SMAs)}

Shape memory alloys (SMAs) are metallic alloys that can memorise their original shape and, after being plastically deformed, return to their original shape on heating above its transition temperature. Their advantages of high energy density, significant recovery stress (e.g. $800 \mathrm{MPa}$ ), high strain (typically $8 \%$ ) and no noise make them promising as embedded actuators for soft robots. However, SMAs show non-linear characteristics, including pseudo-elasticity that is associated with stress-induced phase transitions, shape memory effect and high damping capacity due to their high mechanical hysteresis and internal friction, also associated with its stress-induced phase transitions. The need for a high-temperature control mechanism and long cooling time can restrict their applications due to the low frequency of operation.

Stirling et al. fabricated an active SMA-based soft orthotic, as illustrated in Figure 6a [57]. The active, soft orthotic (ASO) is comprised of four sets of SMA (NiTi) actuators on the dorsal surface of the knee to assist flexion and an SMA set on the frontal surface to assist extension. Each actuator set consisted of four lines of the SMA coils. This design enabled a large deflection angle of $35^{\circ}$ for knee motion and demonstrated the concept of embedding SMA actuators into soft materials for creating soft rehabilitation devices. The ASO was able to provide individualised control since the actuators were able to mimic muscle functionality and assist muscle rehabilitation effectively. However, some key challenges remain; for example, the response time of the SMA actuators and cooling time are $25 \mathrm{~s}$ and 40-50 s, respectively, which are significantly slower than fluid-powered driven soft actuators.

Lai et al. designed a novel SMA-based splint for finger joints rehabilitation, as shown in Figure 6b [58]. Four wires of a shape memory alloy (SMA - Ti50Ni45Cu5 alloy $1.0 \mathrm{~mm}$ in diameter and $110 \mathrm{~mm}$ in length) were arranged in parallel within silica-gel tubes to form a splint, which is a flexible joint that actuates during rehabilitation when the SMA is controlled by heating and cooling, see Figure 6c. A fixture splint that has an adjustable mechanism to fit a variety of fingers with a PC-based control system was developed for a medical clinic milieu use, while a portable splint controlled by a microcontroller (PIC18F452) was developed for home rehabilitation. The maximum output force of a finger splint was $22 \mathrm{~N}$, which is sufficient for a finger rehabilitation process. However, the need for temperature control during the actuation of the SMAs leads to a long response time of the splint, typically 16-19s.

To effectively assist in hand rehabilitation, Hadi et al. presented a novel lightweight hand exoskeleton robot actuated by SMA-based tendons [59]. A glove was used as the interface between the SMA actuators and human hands, where SMA wires for both proximal and distal tendons of each finger were assembled on the glove. For each finger, two DoFs were actuated via the SMA wires connected to the proximal phalanx and the end of the distal phalanx, as shown in Figure 6d. To avoid mutual interference of SMA wires during actuation, several guides were mounted on the glove for improved connection and transfer of the associated force to the desired phalanxes. When the SMA actuators were activated, their tensile force and length variation could be transformed to the joint angles, as shown in Figure 6e. This glove was able to generate a $90^{\circ}$ angular movement and produce a grasping force of over $40 \mathrm{~N}$, and the speeds of assisting to hand flexion and extension were 3 and 4 seconds, respectively. Kazeminasab et al. improved the performance of this glove by exploiting variable structure controllers, and the optimised glove has the potential for physiotherapy and assistance [60]. During operation in a physiotherapy mode, hand motion and the required joint trajectories are controlled, while in the object manipulation mode, the grasping force is controlled. The maximum output force generated by the glove is approximately $45 \mathrm{~N}$, and the speed of hand flexion and extension are 2.5 and 
4 seconds, respectively. Compared to the initial design [57], the size of the device is still quite large and more compact and portable designs are needed for remote use; for example by exploiting coiled or finer scale SMA materials.

The wrist joint is critical for force transmission between the forearm and the hand, and its stability affects the capability and motion of the hands and fingers [61]. Therefore, wrist rehabilitation aims to restore the normal range of movement of joints and facilitate hand and finger function. Serrano et al. developed an SMAbased soft glove for wrist joint rehabilitation [62]. The device was integrated with a rigid skeleton to provide a range of motion between $-10^{\circ}$ to $30^{\circ}$ and a relatively low response frequency of $0.04 \mathrm{~Hz}$. A PID controller was designed to effectively control motion, however the control of non-linearly requires more investigation. The device weighed $960 \mathrm{~g}$ and was low noise, which is suitable for rehabilitation. Villoslada et al. proposed a soft wearable prototype for wrist joint rehabilitation based on flexible NiTi SMA actuators [63]. The SMA wire was placed into the interior of a Bowden cable sheath made of nylon. When the SMA wire was actuated, an extension of the wrist was achieved. The SMA actuator was designed to be bent at angles up to $180^{\circ}$, providing free movement and improved integration with wearable robotic devices. In addition, PID control algorithms were used for velocity and position control of the SMA, which enhanced its actuation bandwidth and increased its service life by preventing overheating during operation.

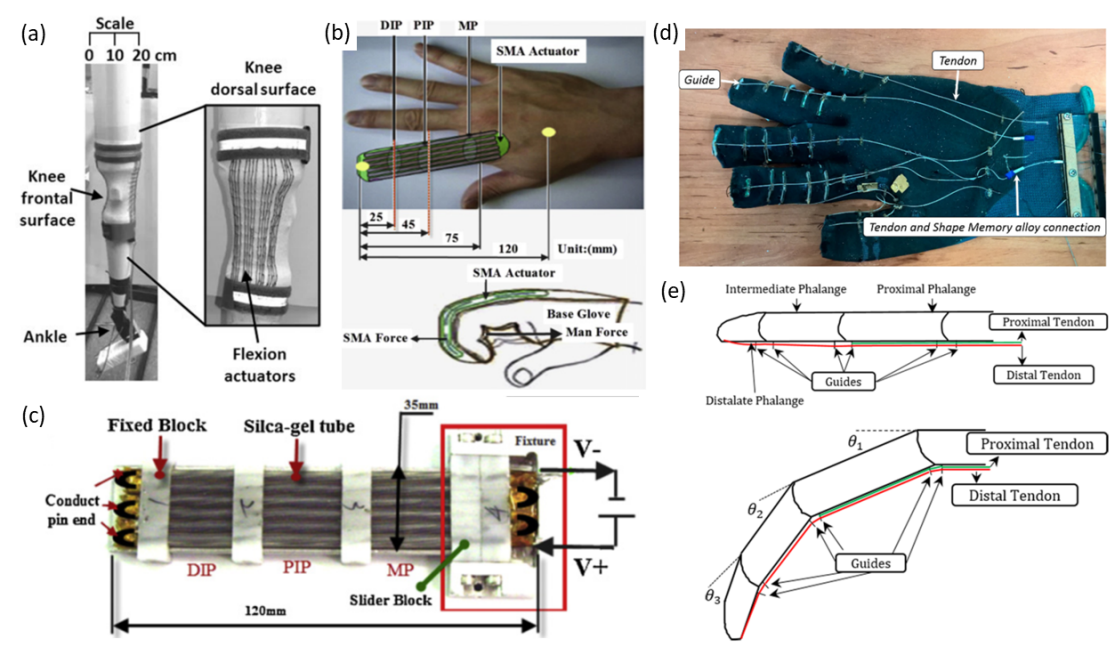

Figure 6: Rehabilitation and assistive devices using shape memory alloy (SMA) for knees and hands. (a) An active, soft orthotic (ASO) for the knee using SMA springs [57]. (b) Concept and (c) finger glove of the SMA-based dynamic splint [58]. (d) Light-weight hand exoskeleton robot actuated by SMA tendons and (e) operation mechanism [59].

An ankle-foot orthosis (AFO) is an assistive device for improving the function of the foot and ankle joint [66]. Passive AFOs apply springs and dampers to provide motion control during the human gait, but they generally create excessive resistance to plantarflexion in the stance phase, thereby restricting ankle motion and disturbing stability of the leg. Since SMA-based active AFOs have the potential to improve AFO performance, Mataee et al. designed two SMA-based AFOs by taking advantage of their super-elasticity [67]; this is a phase induced phase transformation that provides large elastic deformations. In the first design, an SMA rod was used to provide variable torsional stiffness by exerting an axial preload, while in the second design, the bending stiffness of the SMA element was controlled. As shown in Figure 7a, the superelastic element was designed as a hinge of the articulated orthosis. The linear actuator was able to control the vertical position of the slider, which determines the active length of the SMA hinge, resulting in the variation of the bending stiffness, which benefits the AFO. Sadeghian et al. also designed a knee-ankle-foot orthosis with stance and swing for patients with quadriceps muscle weakness that operate SMAs, see Figure 
$7 \mathrm{~b}$ [68]. The orthosis is needed to maintain the stability of the knee joint during the stance phase and assist in the extension of the leg using SMAs during the swing phase. The swing phase mechanism was based on an SMA, and a cantilever SMA beam was subjected to a bending load during flexion, and the extension motion of the knee joint was achieved by releasing the stored energy in the beam, exploiting the super-elastic properties of SMA. For correcting the walking gait for patients, a longitudinal slider was designed at the end of the SMA beam to adjust the stiffness of the SMA element by varying its effective length. The return torque of the SMA bending element was controlled by the walking speed.

To enhance the performance of SMAs and their controllability, Hau et al. developed an ankle rehabilitation device using SMA wires with a periodic cooling system to provide foot plantarflexion and dorsiflexion during rehabilitation exercise, see Figure 7c [69]. The device consisted of eight aluminium metal plates, a plastic shaft, an aluminium cylinder, a rubber ring, and an aluminium connector. The SMA wire was coiled around the aluminium metal plates, which also act as a cooling system to rapidly cool the SMA wires to improve the speed of response. Four conventional on-off fans were also used to enhance the cooling rate of the SMA wires, producing an airflow of $61 \mathrm{~m} 3 / \mathrm{h}$ at $3500 \mathrm{rpm}$ from each fan. The periodic cooling system for SMA wires provided an excellent heating/cooling cycle time of only 5.7 seconds. Most of the SMA actuators presented above use SMA elements with large length-diameter aspect ratios, such as wires, beams, and rods. The deformation of these shapes is mainly in the form of elongation, bending or twisting, which could result in stress concentrations and mechanical failures on the SMA materials, including fatigue. As an alternative approach, Pittaccio et al. created a novel hinge-shape SMA that can be readily applied to the rehabilitation of the elbow and ankle joint, see Figure 7d [70]. Inside the hinge-shaped SMA actuator, an SMA spring was inserted that was shaped like a capital letter omega $(\Omega)$. When loaded, the actuator was compressed, and the output force could reach up to $30 \mathrm{~N}$. The hinge-shaped SMA can be trained by using thermo-mechanical treatments in the shape setting stage to suit patients' needs and provide more personalised characteristics.

Since soft assistive devices have the potential for daily use in the home and to assist the elderly for staircase climbing, Joudzadeh et al. [71] proposed a conceptual design of an SMA-based lightweight wearable device, see Figure 7e. A tendon driven system was used for motion and as illustrated in Figure $7 \mathrm{f}$, the length of the SMA tendon varied when a rotation of the knee angle was produced. Motion during staircase climbing can be divided into two stages: in the first stage, a constant torque was applied at the knee joint to make the concept device move up the stair; in the second stage, a tendon system was substituted in the concept device, and a constant force was applied to the knee-tendon to create the same torque of the first stage. The simulated results show the feasibility of using SMA wires as actuators for this form of assistive device. However, the time for cooling SMA wires is approximately $90 \%$ of the climbing cycle, therefore an efficient cooling system is needed to achieve a shorter cycle time. To assist the upper limb muscular strength, Park et al. proposed a SMA-based fabric muscle (SFM) which is placed in a wearable arm suit, see Figure $7 \mathrm{~g}$ [72]. When a wearer lifts an object and moves, the SFMs contract and assist the muscular strength of the arms and the contraction length of the SFM can be regulated to operate at a range of conditions. A position controller for the length of the SFM was developed, in which a K-type thermocouple was used to monitor the SFM's temperature and control the input current for Joule heating. While the wearable device weighs less than $1 \mathrm{~kg}$, it can lift over $4 \mathrm{~kg}$ in weight. In addition, the response time of moving to the target position was only 3 seconds, but the required relaxation time during cooling is approximately $30 \mathrm{~s}$. Therefore, an additional cooling system can further enhance the performance of the soft arm suit.

Shape memory alloys (SMAs) wires and springs are gradually used in the field due to their high energy density, large recovery stress (e.g. $800 \mathrm{MPa}$ ), high strain (typically $\sim 8 \%$ ), lightweight nature and zero noise. $\mathrm{Ni}$-Ti based alloys is widely used as the material. Current SMA-based rehabilitation and assistance devices include soft knee, ankle and foot orthoses [57,67, 69-71], hand, finger and elbow assistive devices [58-60, 70], soft gloves for wrist joint rehabilitation [62, 63], soft devices for climbing assistance [71] and muscle strength assistance [72]. They provided force in the range of $10 \mathrm{~N}-100 \mathrm{~N}$. However, since they require changes in temperature to produce phase changes of the alloy they present a long response time (10s - 30s) and significant nonlinearity, resulting in the challenge in the design of control systems. The state-of-the-art devices use PID controllers [62-63, 68], adaptive control systems [31, 33] and Bluetooth $\AA$ tracing technology 

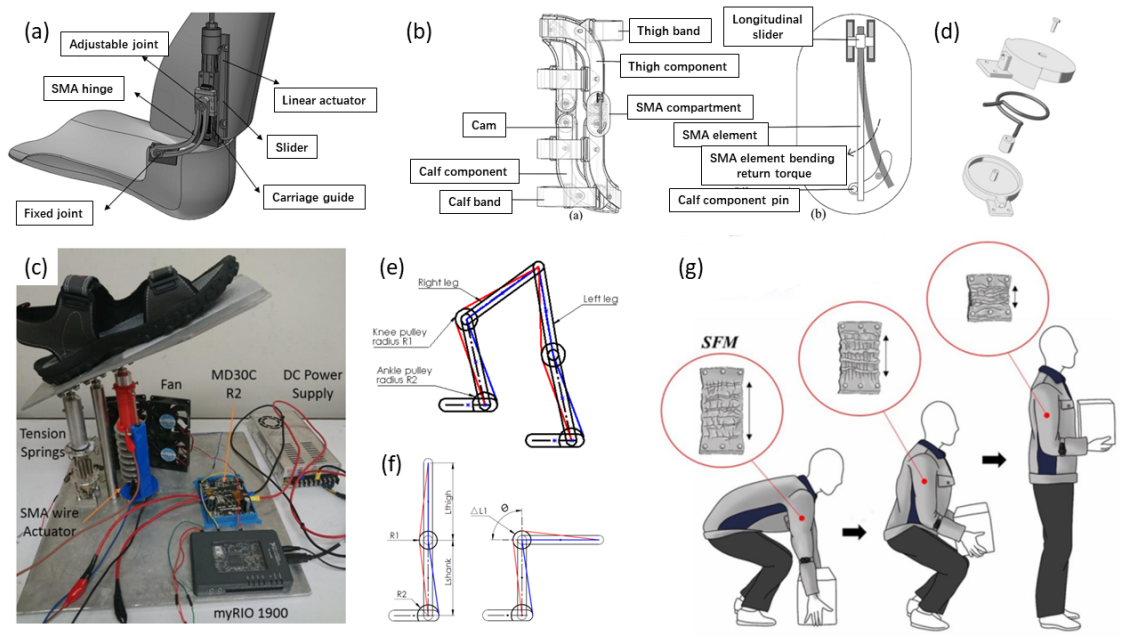

Figure 7: Rehabilitation and assistive devices using shape memory alloy (SMA) for lower limbs and weightlifting. (a) Ankle-foot orthosis (AFO) based on the super-elastic SMA hinge [67]. (b) Knee-ankle-foot orthosis for patients with quadriceps muscle weakness [68]. (c) Ankle rehabilitation platform with a periodic cooling system [69]. (d) Novel omega $(\Omega)$-shape SMA actuator for neurology and neuromuscular rehabilitation [70]. (e) Concept of an SMA-based wearable robotic assistive device for stair climbing assistance and (f) mechanism [71]. (g) SMA-based fabric muscle (SFM) used for suit-type wearable robot (STWR) [72].

[70] in order to achieve desired motions. The control of SMAs needs to be further investigated to enhance the capabilities of the soft devices for better assistance. SMA-based soft rehabilitation and assistive devices are still in their infancy. Research in the development of SMA materials, fundamental understanding in material properties, control and sensing methodologies need to be explored for the potential of SMA-based assistive devices for patients' rehabilitation and assistance of people's daily activities.

\section{Dielectric elastomers actuators (DEAs)}

Dielectric elastomers actuators (DEAs) have been used for soft actuators and robots because of their advantages of large strain and high compliance. Currently, commercially available acrylate-based (e.g., VHBTM) polymers are often used for DEAs because they exhibit large area strains of up to $380 \%$ and possess relatively high dielectric constants $(? \mathrm{r} \sim 4.7)$ and breakdown field strengths [73]. Since Young's modulus of dielectric elastomers is in the range of biological tissues, rehabilitation and assistive devices that aim to exploit DEAs can provide safe human-device interaction. DEAs also have good muscle-like behaviour, which can mimic the behaviour of skeletal muscles. Lidka et al. studied the feasibility of using a $40 \mu \mathrm{m}$ thick silicone-based DEA as an actuator within a wearable assistive device for wrist rehabilitation [74]. The DEA was sprayed with a graphite powder to act as a stretchable electrode, which was tested for its capabilities for wrist rehabilitation in terms of force and range of motion. The results showed that this single layer DEA is insufficient to actuate an assistive wearable wrist rehabilitation device, as the maximum output force is only $0.93 \mathrm{~N}$. Therefore, a DEA device based on multiple layers was recommended to enhance the device output performance. Behboodi et al. investigated the electromechanical properties of DEAs by assessing their capabilities and limitations for the use in robotic rehabilitation devices [75]. A commercial stacked DEA manufactured by CTSystem [76] was used for investigating the concept. The strain of this stacked DEA device was $3.3 \%$, which is lower than human skeletal muscles of $20 \%$. While the maximum cycle life of mammalian skeletal muscles is 1000 million cycles, the cycle life of stacked DEA is 50 million cycles, which is considered sufficient for rehabilitations.

Increasing interest of DEA-based soft rehabilitation and assistive devices has focused on the improvement of 
the output strain and force, along with the reduction of the size. Duduta et al. developed a stacked DEA to actuate elbow rehabilitation robotic devices, as shown in Figure 8a [77]. This soft composite DEA combines a UV-curable strain-stiffening elastomers with ultra-thin carbon nanotube (CNT)-based percolative electrodes. Compared with conventional carbon grease electrodes, the CNT electrodes can help increase the dielectric breakdown field, leading to operation at high electric fields and therefore higher strain or force. Since the output force of a single-film DEA is usually small, they used a multi-layer structure to achieve the desired forces $(>10 \mathrm{~N})$ and displacements $(>1 \mathrm{~cm})$. The stacked DEA demonstrated a peak energy density of 19.8 $\mathrm{J} / \mathrm{kg}$, which is close to the upper limit for natural muscle $(0.4-40 \mathrm{~J} / \mathrm{kg})$. Carpi et al. [78] proposed the concept of using a folded DEA to optimise the dynamic hand splints for finger rehabilitation, see Figure 8b. The elastic band in conventional dynamic hand splints is substituted with a folded DEA to achieve active control. The folded DEA was fabricated using a silicone substrate and a silicone/carbon black stretchable electrode. As shown in Figure 8c, the folded DEA contracts when actuated by an applied potential and pulls up the finger; when the actuation signal is removed, the folded DEA returns to its original state. The electrical activation of the DEA is utilised to control the compliance of the system, enabling the regulation of the force against finger movements. Behboodi et al. developed an elbow joint rehabilitation robotic device actuated by a $3 \times 5$ DEA array, see Figure $8 d$ [79]. The $3 \times 5$ DEA muscle was directly attached to the forearm of a phantom model. The resulting DEA array was able to significantly improve the driving force of artificial muscles up to $30.47 \mathrm{~N}$ and provide $19.5^{\circ}$ of elbow flexion and $16.2^{\circ} / \mathrm{s}$ angular velocity for the elbow, under a $1 \mathrm{~N}$ tensile load.

To reduce the size of actuators, Saharan et al. [80] utilised Twisted and Coiled Polymer (TCP) muscles to assemble 3D printed hand orthosis for facilitating the motion of hand joints, see Figure 8e. The TCP muscles were fabricated using silver coated Nylon 6,6 threads. The soft orthosis consisted of an arm attachment, stitched fabric for the wrist and the palm with guideways, and three rings per finger with one for each of the three finger joints. The TCP muscles were actuated by an electrothermal field and could achieve temperatures up to $250^{\circ} \mathrm{C}$ during actuation. A thermal protection layer below the TCP muscles was designed for the safety of the user. The orthosis was capable of restoring basic human hand movements, such as flexion and extension. While the device weighed only 90 grams, it was able to grasp everyday use items. Amin et al. [81] proposed a concept of a soft hand rehabilitation device, as shown in Figure 8f, which was actuated by a spring-roll DEA that provided the advantages of small size, simple structure and ease of control, as shown in Figure 8g. The DEA was made of VHB 4910 elastomer sheets and carbon conductive grease electrodes. The application of a pre-strain to the dielectric elastomer was able to improve the actuator performance and the breakdown strength [82]. A metallic screwed core was used to compress the spring before wrapping the pre-stretched dielectric elastomers. The spring roll DEA was small and easy to control and the output axial forces under activated and deactivated status were $12.66 \mathrm{~N}$ and $14.71 \mathrm{~N}$, respectively.

Li et al. designed a wearable assistive device for the hip joint during walking using a plasticized polyvinyl chloride (PVC) gel and meshed electrode-based DEA [83]. The device had a control and power system for outdoor walking, as shown in Figure 9a, and exhibited biological muscle behaviour with high deformation $(>10 \%)$, high stress $(>90 \mathrm{kPa})$ and variable stiffness. A multi-layer structure was introduced to increase the output force. When actuated, the DEA contracts and generates a moment on the hip joint to move the thigh forward, see Figure 9a. The maximum output force was $94 \mathrm{~N}$, and the weight of the device was $2 \mathrm{~kg}$. In a preliminary evaluation, a hemiparetic stroke patient successfully performed a $10 \mathrm{~m}$ to-and-fro straight-line walking task at a walking speed of $0.8 \mathrm{~m} / \mathrm{s}$. This assistive device facilitates the natural movement of the patient, increases the step length, and decreases muscle activity; it also has the advantages of a lightweight nature, fast response time, and low drive voltage $(400 \mathrm{~V})$.

In addition to mimicking the contractive behaviour of skeleton muscles, DEAs are also able to provide a high compression pressure. Disorders associated with the lower extremity venous system significantly affect the quality of life of people. These disorders often cause orthostatic hypotension, oedema, deep vein thrombosis and other conditions related to insufficient venous blood return. A DEA-based active compression bandage (ACB) for assisting the human lower extremity venous system was designed, prototyped and tested by Pourazadi et al. [84]; see Figure 9b. A customised calf prototype was developed to measure the pressure applied 

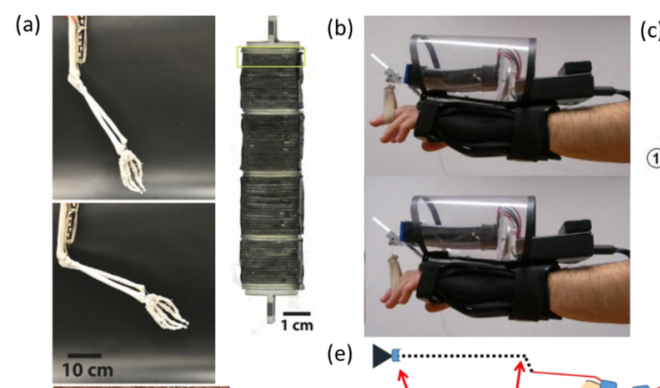

(d)

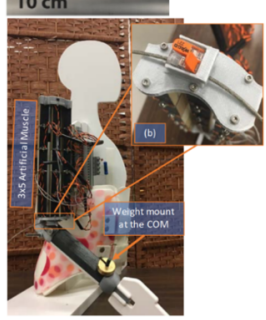

(e)

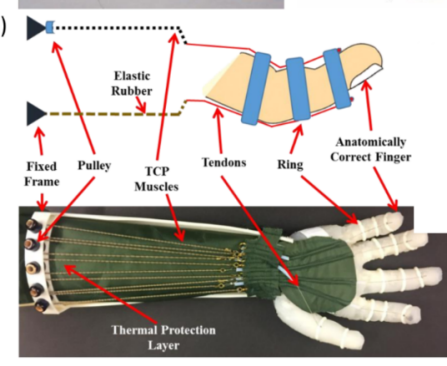

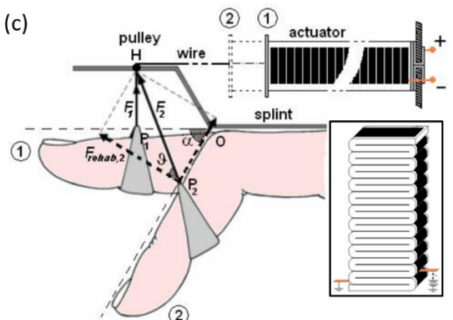

(f)

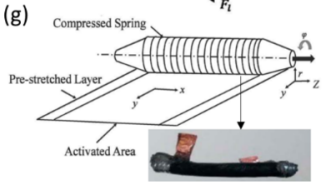

Figure 8: Rehabilitation and assistive devices using dielectric elastomers materials for upper limbs. (a) Elbow rehabilitation robots actuated by a stacked dielectric elastomer actuator (DEA) [77]. (b) Hand splints for finger rehabilitation and (c) mechanism [78]. (d) Elbow joint rehabilitation device actuated by a $3 \times 5$ DEA array [79]. (e) Hand assistant DEA-based soft skeleton actuated by Twisted and Coiled Polymer (TCP) muscles [80]. (f) Concept of a hand rehabilitation system actuated by a spring roll DEA and (g) mechanisms and prototype [81].

by the compression of DEAs fabricated using silicone substrates and copper electrodes. A finite element model (FEM) was developed to simulate the electromechanical behaviour of the DEA. The compression range of the ACB was approximately $15-20 \mathrm{mmHg}(2000-2670 \mathrm{~Pa}$ ) from knee to ankle, which is similar to the mildest grade of compression stockings. The relationship of the actuation pressure $(\mathrm{Pa})$ and the applied electric field for ACBs at different calf regions is shown in Figure 9c.

The large strain, high compliance and fast response of DEAs provide the advantages for the development of soft rehabilitation and assistive devices. However, most of DEA-based devices remain in the concept stages and there are few prototypes for real-life use. This is because the drive mechanism for DEAs normally needs a high electric field, and therefore drive voltage, which can cause human-device interaction safety issue, in addition to the bulky size of the devices. Since the actuation material is a polymer the force provided by an individual DEA $(1-10 \mathrm{~N})$ is normally not sufficient to assist or support human movements. A variety of DEA structures, such as stacked and multilayer DEAs [75, 77, 83], folded DEAs [78] and a spring-roll DEA structure [81] are used to realise larger output force. For example, a maximum output force of a PVC gel-based wearable assistive device with a multilayer DEA structure can achieve $94 \mathrm{~N}$, which can support the hip joint. However, it was high in weight and bulky in terms of size. To bring DEAs to the real assistive devices, there are four key challenges (i) the safety consideration of using high electrical field, and high drive voltages, when actuating DEAs. (ii) the need of compactness for the devices (iii) the improved performance of DEAs to generate larger force (iv) the current state-of-the-art DEA-based devices are based on feedforward control. Effective, efficient and robust control systems are needed to control the devices to suit rehabilitation and people's needs.

\section{Magneto-active elastomers (MAEs)}

A magneto-active elastomer (MAE) is a composite made from an elastomeric matrix and magnetisable fillers [85]. In the presence of a magnetic field, soft MAEs are able to induce elongation, contraction, bending, and 
(a)

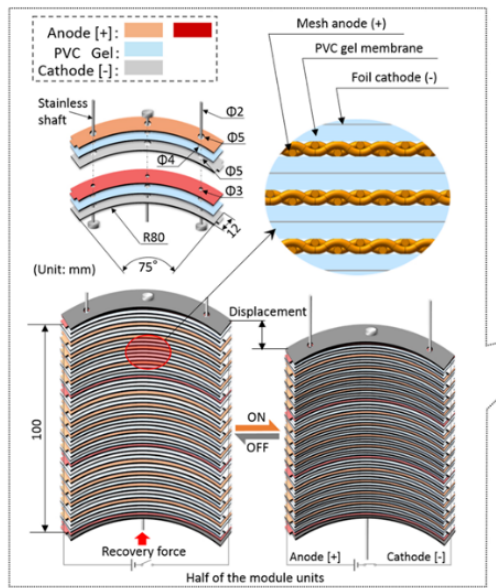

(b)
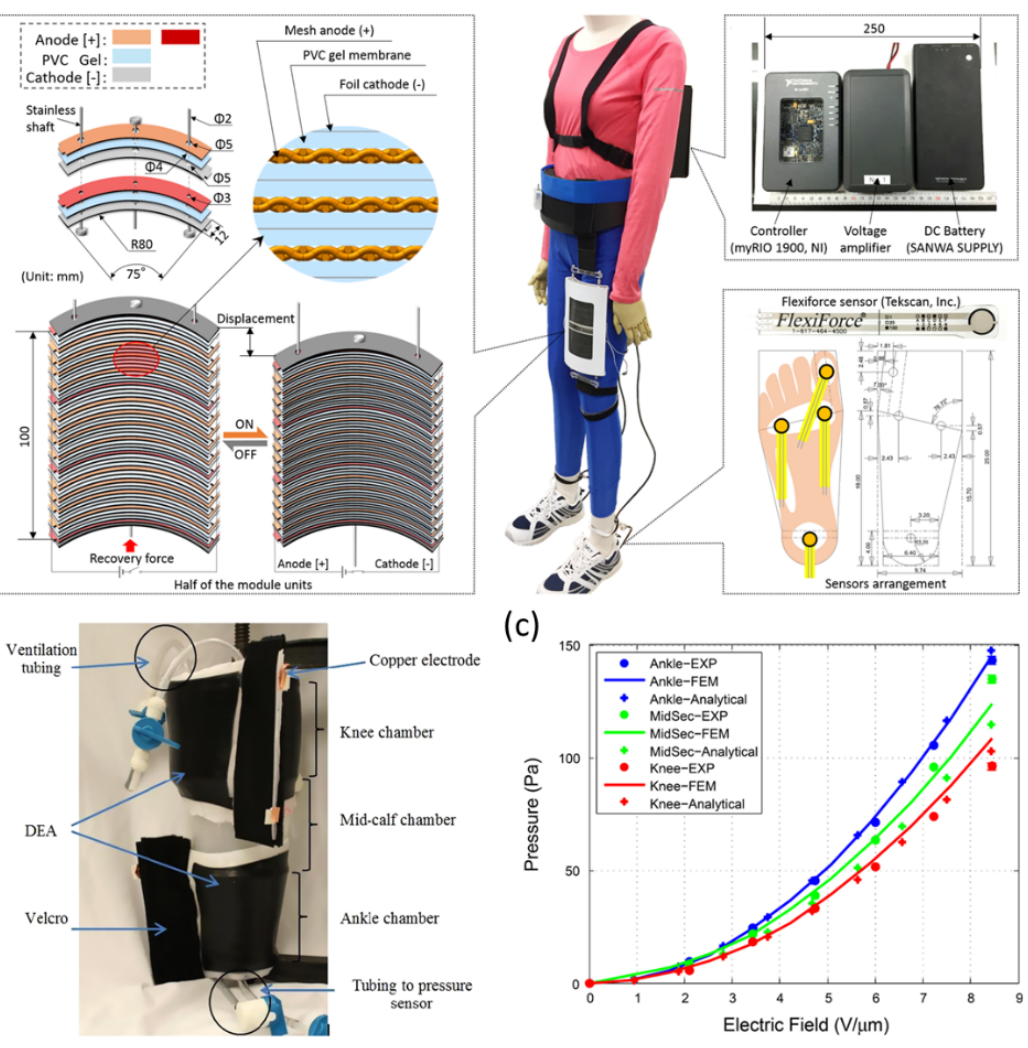

(c)

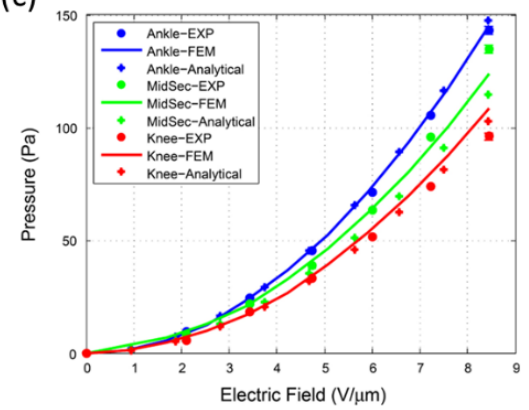

Figure 9: Rehabilitation and assistive devices using dielectric elastomers materials for lower limbs. (a) dielectric elastomer actuator (DEA) based wearable assistive device for hip joint rehabilitation [83]. (b) DEA-based active compression bandage (ACB) and (c) the relationship between the applied electric field for ACBs at different calf regions [84].

a range of other modes of deformation [86]. The magnetisation profile, actuation signal, and overall shape of filler materials are the major parameters that affect MAE deformation. To date, MAEs have been developed for soft robots targeted for biomedical devices and drug delivery, while there are fewer reports of MAE-based devices for rehabilitation and assistance due to the relatively low output force, actuation mechanism and control strategies. Nadzharyan et al. proposed a concept of a retinal fixator for retinal detachment surgery using MAEs, which consisted of a silicone elastomer matrix and embedded magnetic iron macroparticles [87]. The elastic modulus of the MAE was $104 \mathrm{kPa}$, while the stress and relative strain at break was $0.3 \mathrm{MPa}$ and $141 \%$, respectively. The fixator applies an MAE, which is used as a patch that is placed inside the eye on the retina surface to act as a seal. Permanent magnets covered with medical silicone are attached to the sclera to locate and fix the inner MAE patch, as shown in Figure 10a. The two components can be closely attached using the magnetic force to fix the retina to the underlying tissues. The relationship between the attraction force and their separation distance in a flat 'patch-magnet' system was studied by Finite Element Analysis and experimental validation. An enhanced theoretical model which includes the 'patch-magnet' system with a range of possible geometric configurations was also developed and used for investigating the effects of the change in the geometry [88][89]. The results showed that small changes in the geometry have strong effects on the interaction force and magnetic pressure. Figure 10b shows the relationship of an average pressure achieved from the measured interaction force and the surface area of the MAE sample with the distance between an MAE and the magnets. Force measurements were performed for both flat and curved configurations. The results showed that small size systems can produce considerable attraction force and 
can be potentially applied for retinal detachment surgery. It will be of interest to exploit such MAE force generation and fixture mechanisms to other soft systems.
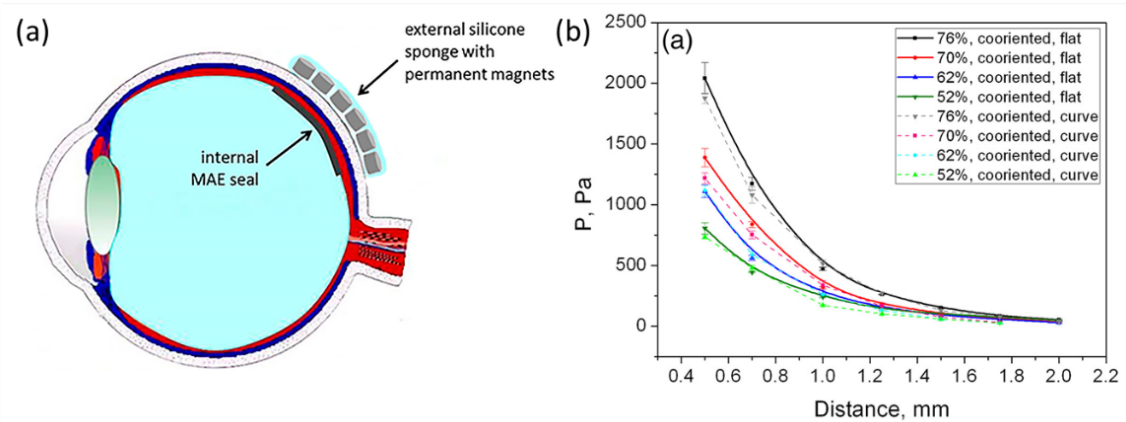

Figure 10: Magneto-active elastomer-based retina fixator and the relationship between average measured pressure and the distance between a magneto-active elastomer and the magnets [89].

Soft MAEs can perform a range of modes of deformation, including elongation, contraction and bending with a magnetic field. They are highly promising in medicine and healthcare, such as drug delivery, biomedical applications and disease treatment. However, to date, there are few applications in rehabilitation and assistance since the output force is small and the actuation systems need to be designed to be less bulky and more versatile in their deformation.

\section{Liquid crystalline elastomers (LCEs)}

Liquid crystalline elastomers (LCEs) are materials that combine the entropy elasticity of polymer elastomers with the self-organisation of liquid crystals [90]. LCEs can reversibly change shape to generate motion in response to a range of stimuli such as temperature, $\mathrm{pH}$ variations, light, electric, or magnetic fields, which enables them to be used as soft actuators. Ferrantini et al. reported a biocompatible acrylate-based lightresponsive LCE that can be activated continuously for long periods of time with a low light intensity within a physiological environment [91]. The materials are able to act as artificial muscles and reproduce the cardiac twitch contractions. The mechanical properties of the LCE can also be modulated by light intensity, stimulation frequency and duty cycle to fit different contraction amplitude/time courses of human muscle. To prove this concept, actuation tests were performed on a mouse muscle. The results showed the LCEs can improve muscular systolic function, with no impact on diastolic properties, which indicated LCEs are promising in assisting cardiac mechanical function and developing a new generation of contraction assist devices.

LCEs are new to the field of soft rehabilitation and assistive devices, and the actuation mechanism is more complex than other systems such as fluid-powered and electrical driven systems. They can reversibly change shape to generate motion in response to a range of stimuli such as temperature, $\mathrm{pH}$ variations, light, electric fields, or magnetic fields. However, the dynamics and responses of LCEs are difficult to control accurately. The state-of-the-art technology uses a feedforward control mechanism to achieve the desired motion. There are few soft rehabilitation and assistive device prototype in the field, although the concept of the cardiac contraction device [91] is a highly promising start in the field by using LCEs to assist the cardiac treatment and further applications can emerge from improved system design and control.

\section{Piezoelectric materials}

Piezoelectric materials are materials that produce an electric charge when they are placed under mechanical stress (sensor) or provide a strain in response to an applied electric field (actuator). Polyvinylidene fluoride 
(PVDF) is one of the most promising piezoelectric materials for soft robot applications, as it possesses superiority in an ability to be formed in thin-film form, low weight and high mechanical flexibility. However, in actuation mode, the strains are low, the forces capability is small, and they require high driving electric fields. For soft rehabilitation and assistive applications, they are therefore widely used as a sensing element by converting mechanical energy into the electrical signal and show excellent performance such as high sensitivity (volts per applied stress), increased flexibility, lightweight, and self-powered characteristics since a charge is generated by the direct piezoelectric effect which is directly proportional to the level of applied force. Asadnia et al. developed a highly stretchable, self-powered and ultrasensitive strain sensor for fingers using PVDF nanofibers [92]. Using aligned PVDF nanofibers, gold electrodes were fixed on a liquid-crystalline polymer substrate using a conductive carbon tape. A soft assistive glove with two sensors mounted on the middle and index fingers was fabricated for recognition of finger motion. The sensing feedback provided by the soft gloves provided the potential to open up new avenues in rehabilitation engineering and brings a sense of touch to myoelectric limbs. Alluri et al. developed a simple, large-scale fabrication technique to form spherical piezoelectric composite (BaTiO3 nanoparticle/Ca-alginate) beads using an ionotropic gelation method [93]. A high output $(82 \mathrm{~V}, 227 \mathrm{~mA})$ was generated during the application of a low mechanical pressure of 1.70 $\mathrm{kPa}$; in addition to providing a sensing functionality, the voltage/current was reported to be sufficient to drive low power electronic devices. The device made from this novel piezoelectric material was able to act as a self-powered wearable flexion sensor for decoding right arm finger flexion/extension movements. This novel piezoelectric material is non-invasive, robust, cost-effective. It does not require external power for wearable assistive devices, unlike piezoresistive sensors that require an applied current to measure a change of resistance with stress. For gait rehabilitation, Ahmad et al. designed a novel PVDF pressure sensor based on d33-mode polarisation for sensing gait motion, where d33 corresponds to a force applied in the polarisation direction of the piezoelectric material [94]. In contrast to the d31-mode of polarisation, where the materials is polarised in the thickness direction, the d33-mode of polarisation applies the electric field between two conductors and polarised laterally. A round-shaped prototype with a diameter of $15 \mathrm{~mm}$ was able to achieve a sensing sensitivity of approximately $1.85 \mathrm{mV} / \mathrm{kg}$ for load ranging from $1 \mathrm{~kg}$ to $5 \mathrm{~kg}$. On increasing the diameter to $20 \mathrm{~mm}$, the sensor could be used for a load between $38 \mathrm{~kg}$ to $92 \mathrm{~kg}$, which is suitable for gait rehabilitation system. Rajala et al. developed a piezoelectric polymer film in-sole sensor for plantar pressure distribution measurements. The sensor was made of PVDF with evaporated copper electrodes to collect the piezoelectric charge [95]. A $200 \mathrm{~nm}$ thick film of copper was evaporated on both sides of the PVDF substrate using a mechanical mask to produce the sensor electrode pattern. The sensor consisted of eight measurement locations: hallux, first metatarsal head (under both sesamoid bones), metatarsal heads 2-5 and heel. The average peak-to-peak pressure ranged from $58 \mathrm{kPa}$ to $486 \mathrm{kPa}$ and the sensitivity was approximately 28.5 $\pm 1.0 \mathrm{pC} / \mathrm{N}$. The sensor can be used for rehabilitation and sport monitoring for its long-term and costefficient plantar pressure measurement. To identify gait patterns for lower limb rehabilitation, Ma et al. built an intelligent perception system with multiple sensors that included (i) surface electromyography (EMG) sensors for detecting human intention, (ii) photoelectric encoders for determining the angle of hip and knee joints, and (iii) a piezoelectric thin-film sensor to assess the interaction force between the body and the exoskeleton [96]. The interaction forces of both thigh and calf could be measured, and the results showed that the correct rate for gait recognition is up to $92 \%$. Compared to the conventional method that detects gait patterns using a ground reaction force, the new gait recognition system was easy to fabricate and could be used for soft lower limb rehabilitation devices.

Flexible piezoelectric materials are often used as sensing elements in the applications of rehabilitation and assistance since the power levels generated by ferroelectric polymers are relatively low. Due to their need for high electric fields for actuation, it would be highly changeling to apply them as self-powered soft actuators for soft assistive devices directly; their low strain and stiffness also limits their use in actuation. However, the integration of flexible piezoelectric sensing elements with other actuation mechanisms is highly promising for developing intelligent soft rehabilitation and assistive devices. 


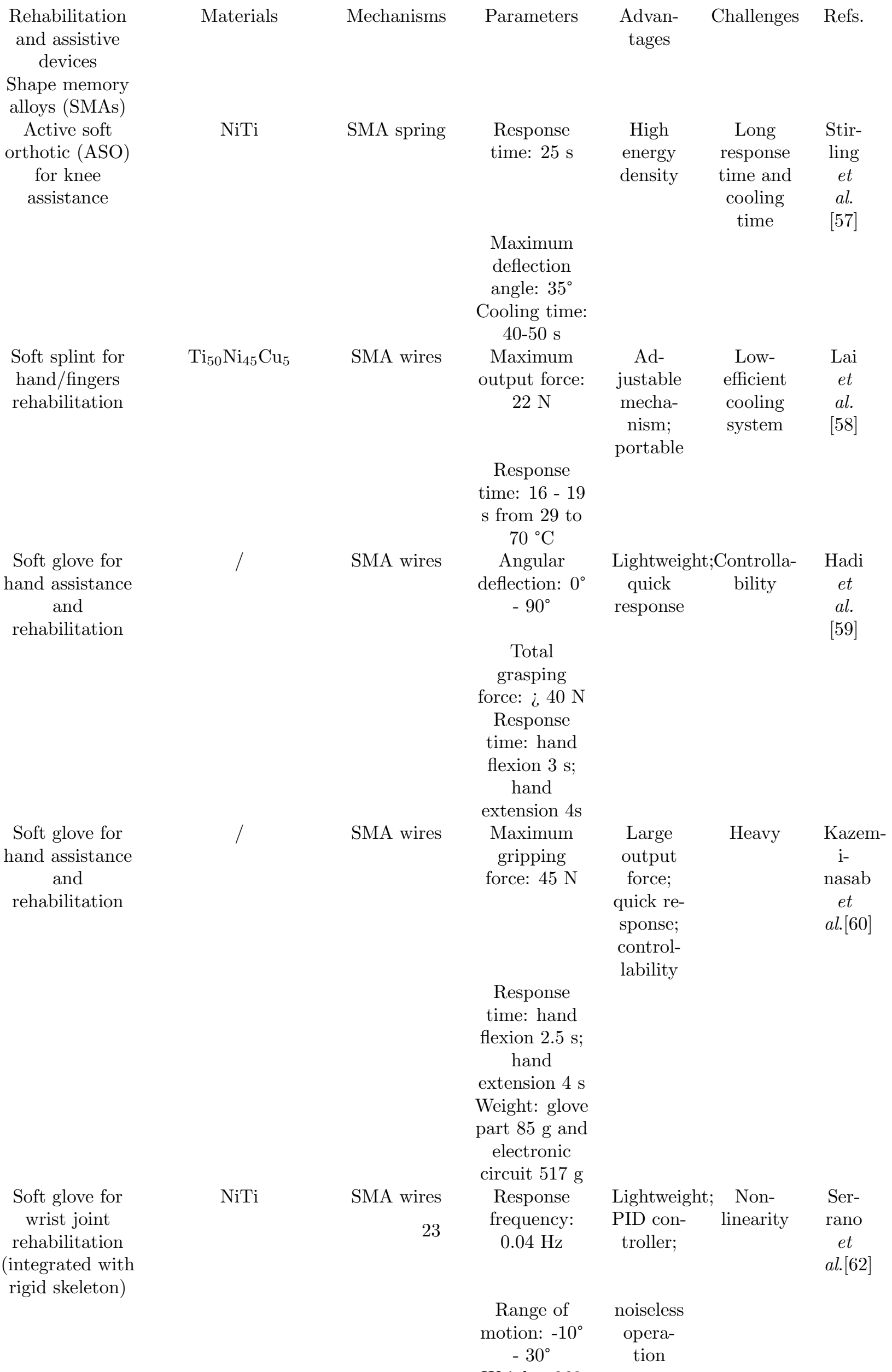




\section{Rehabilitation and assistance enabled by chemical reactions}

The use of chemical reactions is new to soft actuators and robotic devices for rehabilitation and assistance, and the development of rehabilitation and assistive devices based on this intriguing actuation mechanism is still in its infancy. Technical challenges remain in the aspects of actuation output energy, safety and designs related to human-interaction devices. Hosono et al. developed a metal hydride (MH) alloy which can absorb and release 1000 times as much hydrogen gas as its own volume [97]. The reversible chemical reaction is based on an alloy (M) that absorbs hydrogen (H2) by forming a metal hydride (MHx), with the release of heat as shown in equation (1):

$$
\left(\frac{2}{x}\right) M+H 2 \leftrightarrow\left(\frac{2}{x}\right) M H x+Q
$$

where $\mathrm{Q}$ is the quantity of heat involved in the reaction. The hydrogen pressure changes depending on the hydrogen content and temperature to allow actuator control. The conversion mechanism between thermal energy and mechanical energy of a typical MH alloy is shown in Figure 11a.

The reaction can proceed at a constant hydrogen pressure (plateau pressure), and a constant temperature. The metal hydride actuator consisted of two soft bellows which are made from laminated casted polypropylene film (CPP), aluminium (Al) and polyester (PET) films and two hydrogen supplies with metal hydride alloys [97]; see Figure 11b. The total thickness of the laminated film is approximately $0.1 \mathrm{~mm}$. The soft bellows can realise flexion and extension motion by heating and cooling the metal hydride alloys, which exhibit high impermeability to hydrogen gas, flex durability and sufficient passive compliance. Taking these advantages, a toe joint rehabilitation device was developed using metal hydride actuators [98-99], as shown in Figure 11c. The actuator was able to achieve a maximum output force of approximately $200 \mathrm{~N}$ and a maximum velocity of $50 \mathrm{~mm} / \mathrm{min}$ with a load of $5 \mathrm{~kg}$. It can also operate 3500 cycles at $30 \mathrm{~mm} / \mathrm{min}$ with a $2.5 \mathrm{~kg}$ load. A MH alloy used for a toe joint device was able to operate in the range of $0.1-1.0 \mathrm{MPa}$ at a temperature of $20-80^{\circ} \mathrm{C}$. The high impermeability to hydrogen gas of the soft bellows was examined by filling with hydrogen gas and monitoring the pressure change. The results showed approximately $0.7 \%$ pressure depression in the bellows after 240 hours, which validated the high impermeability of the materials used to construct the device. The device driven by the heating and cooling mechanism can provide gentle and slow motion of the toes, which is both human and environment friendly.

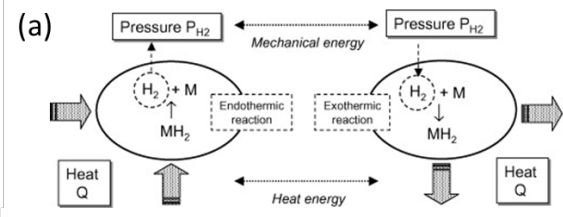

(b)

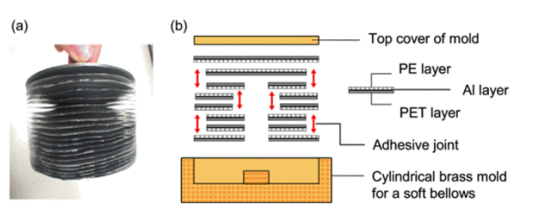

(c)

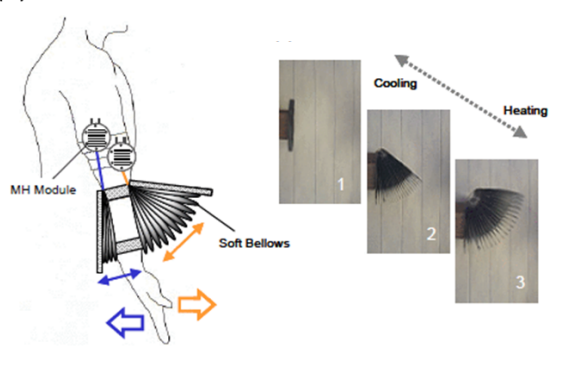

Figure 11: Rehabilitation and assistive devices using chemical reactions for upper limbs. (a) metal hydride $(\mathrm{MH})$ alloy-based laminate bellows and its fabrication mold (b) energy conversion mechanism between thermal energy and mechanical energy of an $\mathrm{MH}$ alloy (c) example of using the $\mathrm{MH}$ based actuator for upper limbs assistance and its actuation stages [97-99] 


\section{Conclusions and outlooks}

Soft actuators and robots enabled by fluid-powered, soft active materials, and chemical reactions have been successfully used in rehabilitation and assistance for hands, wrists, ankles, joes, backs, lower and upper limbs. They can provide the advantages of low cost, ease of manufacture, and high actuation performance and have become a research topic of intense research interest in the field of soft robotics. The soft characteristics of actuators and devices make it possible to improve safe interactions between humans and devices. Not only can the soft actuators and robotic devices be used for medical rehabilitation to provide effective and professional treatment, but they can also be used for assisting in daily activities such as walking, lifting and climbing. While the applications of rehabilitation platforms and assistive devices have previously focused on rigid robotic systems, recent advances in soft actuation have opened up new opportunities for creating new soft rehabilitation and assistive devices. This review has described the current state-of-the-art technologies in this area to demonstrate the nexus between materials, mechanisms, actuation, and applications. We have summarized the latest actuation mechanisms using in soft rehabilitation and assistive devices, including pneumatic and hydraulic fluid-powered actuation, electrical motor actuation, chemical reactions and soft active material-based actuation, which includes dielectric elastomers, shape memory alloys, magnetoactive elastomers, liquid crystalline elastomers, and piezoelectric materials. The advantages and challenges of these devices have been discussed and there are a number of opportunities as to make these devices efficient, portable, wearable, and user-friendly, which are now described.

(1) To realise wearable and portable assistive devices, there is a need to develop compact, safe and efficient power supplies, which can be readily integrated into devices and act as a reliable power source. Assistive devices should fulfil critical safety requirements prior to their practical applications on the human body. For fluid power pneumatic and hydraulic systems, the size of the power supplies has limited the development of untethered assistive devices. For electric field-driven systems, the need for high applied voltages has led to safety concerns for the direct use of devices on humans. Although significant effort has been paid to the development of compact, safe and efficient power sources, the range of currently available power supplies is still limited in terms of their sizes and power mechanisms. In this regard, the design and development of compact, high power density and safe power units for assistive devices will be particularly important.

(2) There is a need to design new and effective soft actuators which can be attached, embedded, or integrated into rehabilitation and assistive devices, such as soft wearable suits. The use of a distributed network of soft pneumatic actuators to develop assistive devices for upper and lower bodies is highly promising, in particular for providing a large range of motion. Clothing-based rehabilitation and assistive devices are able to achieve high degrees of freedom and complex movements, benefiting from the direct guidance and attachment to human bodies. Advanced human-machine interaction and interface technologies can further enhance the functionality of the current state-of-the-art devices. More innovative soft actuators will emerge when actuation performance and mechanisms are combined in synergy with advanced materials with tailored properties.

(3) The state-of-the-art control strategies of soft actuators and robots for rehabilitation and assistance represent a technical challenge. Control strategies are relatively simple as it is challenging to develop accurate models of soft systems, as well as human users. Advanced control algorithms and modelling techniques provide opportunities to enable current control systems to provide a rapid and accurate response, a high degree of robustness, and adaptability. In addition, control parameters of such devices are usually determined or adjusted by experience or use a trial-and-error approach for individuals. Automated customised approaches for the determination of the control parameters will be highly useful in the field and therefore artificial intelligence (AI), such as machine learning, can be used for the design of control systems that are tailored to the needs of individuals.

(4) The muscle-like mechanical properties and biocompatibility of soft active materials provide significant opportunities for the development of next-generation soft rehabilitation and assistive applications at a range of scales; ranging from the applications in medical treatment to devices for daily use. The actuation mech- 
anism is one of the challenges for widely applying these materials. For example, high-voltages and electric fields are usually needed for soft dielectric elastomer actuator-based actuators, which can be a safety concern for rehabilitation and assistive devices. However, with the development of new materials, it is believed that suitable and safe actuation and power mechanisms will be developed, which will significantly expand the applications of smart materials in rehabilitation and assistance. Flexible piezoelectric materials such as polyvinylidene difluoride (PVDF) have been successfully used as sensing elements in rehabilitation and assistive devices. The advantages of flexibility, accuracy, and cost-efficiency of PVDF makes it ideally suited for integrating into these devices for motion sensing and detection. With the development of soft rehabilitation and assistive devices, it is believed that flexible piezoelectric materials can be applied more widely in the field.

(5) Soft MAEs and LCEs are very new to the field of soft rehabilitation and assistive devices. There are few applications and prototypes, and the majority of work to date is at the concept stage. However, the advantages of MAEs and LCEs have shown promising results in medicine, biomedical and healthcare. Future research directions can include (i). the design and development of compact, portable MAEs assistance systems for individuals at home. This will expand the areas and provide sustainable development for independent living and healthcare. (ii). the design of advanced control systems to accurately control the rehabilitation and assistance devices to suit individual needs. The state-of-the-art control systems for MAEs and LCEs are based on the feedforward control structure. Effective sensing elements and feedback control systems would result in a breakthrough in the area, which can accelerate the development of assistance devices. (iii). The output force of the MAEs and LCEs are relatively small, compared to the fluid-powered systems. The creation and fabrication of new materials can directly benefit the area for more possible applications, for example, lower and upper limbs rehabilitation and movement support.

(6) The use of chemical reactions is new to soft actuators and robotic devices for rehabilitation and assistance, but it is a highly promising approach as a human and environmentally friendly actuation mechanism. The storage of actuator chemicals is one of the challenges for the implementation on rehabilitation and assistive devices, which require safe and consistent energy supplies. In addition, the control of the chemical reaction and energy release can also be challenging for assistive devices, which requires accurate and robust control to provide a comfortable user experience. However, this is still an emerging area to explore, and should not be limited with regards to the creative and effective design for rehabilitation and assistive devices, which can combine new actuation mechanisms, materials, sensing and control technologies.

(7). Soft rehabilitation and assistive devices are complex and highly dynamic systems. Unlike individual soft actuators and robot, they are a combination of a variety of actuators, sensors and a dedicated control system, which make it difficult to directly model system dynamics and predict device performance. The understanding of people's needs is crucial to the design of the devices. In addition, although soft devices provide benefits of safer human-device interaction, the difficulty in precise control of the devices provides research challenges. The creation of soft intelligent rehabilitation and assistance devices for healthcare treatment and daily activities assistance is rapidly needed.

While soft actuators and robotic devices for rehabilitation and assistance are in their infancy stage, there continues to be significant scope and opportunities for research and development in this emerging new research area. With a broad multidisciplinary research effort in the pursuit of new soft actuators and systems, it can be envisioned that these new emerging fields will achieve exciting applications in the future and continuously improve our quality of life.

\section{Acknowledgements}

We thank the support from The Leverhulme Trust for the Leverhulme Research Fellowship RF-2020-503 4, the UKRI Innovate UK The Sustainable Innovation Fund, No.79502, the University of Bath Alumni Fund F1920A-RS02 and the University of Bath International Funding Schemes 2020. C.Y and X.L thank the 
support from the China Scholarship Council PhD studentship (201706150102) and the China Scholarship Council visiting Scholar fund (202006150085).

\section{References}

[1] S. Bauer, S. Bauer-Gogonea, I. Graz, M. Kaltenbrunner, C. Keplinger, R. Schwödiauer, Advanced Materials. 2014, 26(1), pp.149-162.

[2] D. Rus, M.T. Tolley, Nature. 2015, 521(7553), pp.467-475.

[3] M. Cianchetti, C. Laschi, A. Menciassi, P. Dario, Nature Reviews Materials. 2018, 3(6), pp.143-153.

[4] T. George Thuruthel, Y. Ansari, E. Falotico, C. Laschi, Soft Robotics. 2018, 5(2), pp.149-163.

[5] S. Coyle, C. Majidi, P. LeDuc, K.J. Hsia, Extreme Mechanics Letters. 2018, 22, pp.51-59.

[6] S. Chen, Y. Cao, M. Sarparast, H. Yuan, L. Dong, X. Tan, C. Cao, Advanced Materials Technologies. 2020, 5(2), p.1900837.

[7] A. Chortos, J. Liu, Z. Bao, Nature Materials. 2016, 15(9), pp.937-950.

[8] D. Chen, Q. Pei, Chemical Reviews. 2017, 117(17), pp.11239-11268.

[9] T. Someya, M. Amagai, Nature biotechnology, 2019, 37(4), pp.382-388.

[10] A.J. Bandodkar, J. Wang, Trends in biotechnology, 2014, 32(7), pp.363-371.

[11] W. Zeng, L. Shu, Q. Li, S. Chen, F. Wang, X.M. Tao, Advanced Materials, 2014, 26(31), pp.5310-5336.

[12] A. Nag, S. C. Mukhopadhyay, J. Kosel, IEEE Sensors Journal, 2017. 17(13), pp.3949-3960.

[13] I. Díaz, J.J. Gil, E. Sánchez, Journal of Robotics, 2011. 2011, 759764,

[14] H.S. Lo, S. Q. Xie, Medical Engineering \& Physics, 2012, 34(3), pp.261-268.

[15] N. Aliman, R. Ramli, S. M. Haris, Robotics and Autonomous Systems, 2017, 95, pp.102-116.

[16] C. Y. Chu, R. M. Patterson, Journal of Neuroengineering and Rehabilitation, 2018, 15(1), pp.1-14.

[17] Z. M. Tsikriteas, J. I. Roscow, C.R. Bowen, H. Khanbareh, iScience, 2020, p.101987.

[18] H.R. Lim, H.S. Kim, R. Qazi, Y.T. Kwon, J.W. Jeong, W.H. Yeo, Advanced Materials, 2020, 32(15), p.1901924.

[19] Q. Lyu, S. Gong, J. Yin, J. M. Dyson, W. Cheng, Advanced Healthcare Materials, 2021, p.2100577.

[20] Q. Xu, X. Gao, S. Zhao, Y. N. Liu, D. Zhang, K. Zhou, H. Khanbareh, W. Chen, Y. Zhang, C. Bowen, Advanced Materials, 2021, p.2008452.

[21] V. Sanchez, C. Walsh, and R. Wood, Advanced Functional Materials, 2021, vol. 31, no. 6.

[22] R.H. Gaylord, S. Heights, 1958, U.S. Patent 2,844,126.

[23] J.R.Allen, A. Karchak, R. Snelson, abstract in Mechanical Engineering, 1962, August: 52-53.

[24] T. J. Engen, The Journal of Bone and Joint Surgery. British volume, 1965, 47(3), pp.465-468.

[25] V. L. Nickel, D. L. Savill, A. Karchak, J. R. Allen, The Journal of Bone and Joint Surgery. British volume, 1965, 47(3), pp.458-464.

[26] B. Tondu, Journal of Intelligent Material Systems and Structures, 2012, 23(3), pp.225-253. 
[27] K.A.Shorter, G.F. Kogler, E. Loth, W.K. Durfee, E. T. Hsiao-Wecksler, Journal of Rehabilitation Research \& Development, 2011, 48(4).

[28] Y. L. Park, B. R. Chen, D. Young, L. Stirling, R. J. Wood, E. Goldfield, R. Nagpal, IEEE/RSJ International Conference on Intelligent Robots and Systems, 2011, pp. 4488-4495. IEEE.

[29] C.M. Thalman, H. Lee, IEEE International Conference on Robotics and Automation (ICRA), 2020, pp. 1735-1741. IEEE.

[30] M. Wehner, B. Quinlivan, P.M. Aubin, E. Martinez-Villalpando, M. Baumann, L. Stirling, K. Holt, R. Wood, C. Walsh, IEEE international conference on robotics and automation, 2013, pp. 3362-3369. IEEE.

[31] C. Thakur, K. Ogawa, T. Tsuji, Y. Kurita, IEEE Robotics and Automation Letters, 2018, 3(4), pp.42574264 .

[32] F. Z. Low, R.C. Yeow, H. K. Yap, J. H. Lim, IEEE International Conference on Rehabilitation Robotics (ICORR), 2015, pp. 589-593. IEEE.

[33] R. Ezzibdeh, P. Arora, D.F. Amanatullah, Arthroplasty Today, 2019, 5(3), pp.314-315.

[34] D. Baiden, O. Ivlev, IEEE 13th International Conference on Rehabilitation Robotics (ICORR), 2013, pp. 1-6. IEEE.

[35] O. Ivlev, IEEE International Conference on Rehabilitation Robotics, 2009, pp. 1-5. IEEE.

[36] D. Baiden, O. Ivlev, The 23rd International Conference on Robotics in Alpe-Adria-Danube Region (RAAD), 2014, pp. 1-6. IEEE.

[37] J. Fang, J. Yuan, M. Wang, L. Xiao, J. Yang, Z. Lin, P. Xu, L. Hou, Soft Robotics, 2020, 7(1), pp.95-108.

[38] A.Wilkening, H. Stöppler, O. Ivlev, IEEE International Conference on Rehabilitation Robotics (ICORR), 2015, pp. 729-734. IEEE.

[39] H. Li, L. Cheng, The 32nd Youth Academic Annual Conference of Chinese Association of Automation (YAC), 2017, pp. 860-865. IEEE.

[40] H. K. Yap, B. W. Ang, J. H. Lim, J. C. Goh, C. H. Yeow, IEEE International Conference on Robotics and Automation (ICRA), 2016, pp. 3537-3542. IEEE.

[41] Yap, H.K., Mao, A., Goh, J.C. and Yeow, C.H., 2016, June. Design of a wearable FMG sensing system for user intent detection during hand rehabilitation with a soft robotic glove. In 2016 6th IEEE International Conference on Biomedical Robotics and Biomechatronics (BioRob) (pp. 781-786). IEEE.

[42] Yap, H.K., Khin, P.M., Koh, T.H., Sun, Y., Liang, X., Lim, J.H. and Yeow, C.H., 2017. A fully fabricbased bidirectional soft robotic glove for assistance and rehabilitation of hand impaired patients. IEEE Robotics and Automation Letters, 2(3), pp.1383-1390.

[43] K. Shiota, S. Kokubu, T. V. Tarvainen, M. Sekine, K. Kita, S. Y. Huang, W. Yu, Robotics and Autonomous Systems, 2019, 111, pp.20-30.

[44] F. Cho, R. Sugimoto, T. Noritsugu, X. Li, IOP Conference Series: Materials Science and Engineering, 2017, 249(1), p. 012004. IOP Publishing.

[45] T. Abe, S. Koizumi, H. Nabae, G. Endo, K. Suzumori, IEEE International Conference on Soft Robotics (RoboSoft), 2018, pp. 572-578. IEEE.

[46] T. Abe, S. Koizumi, H. Nabae, G. Endo, K. Suzumori, N. Sato, M. Adachi, and F. Takamizawa, IEEE Robotics and Automation Letters, 2019, 4(3), pp.2532-2538.

[47] B. W. Ang, C.H. Yeow, 2nd IEEE International Conference on Soft Robotics (RoboSoft) 2019, pp. 577-582. IEEE. 
[48] P. Polygerinos, Z. Wang, K. C. Galloway, R. J. Wood, C. J. Walsh, Robotics and Autonomous Systems, 2015, 73, pp.135-143.

[49] P. Polygerinos, P., Galloway, K.C., Savage, E., Herman, K. O’Donnell, C. J. Walsh, IEEE international conference on robotics and automation (ICRA), 2015, pp. 2913-2919. IEEE.

[50] A. T. Asbeck, S. M. De Rossi, I. Galiana, Y. Ding, C. J. Walsh, IEEE Robotics \& Automation Magazine, 2014, 21(4), pp.22-33.

[51] Y. Mengüç, Y. L. Park, H. Pei, D. Vogt, P. M. Aubin, E. Winchell, L. Fluke, L. Stirling, R. J. Wood, C. J. Walsh, The International Journal of Robotics Research, 2014, 33(14), pp.1748-1764.

[52] A. Atalay, V. Sanchez, O, Atalay, D. M. Vogt, F. Haufe, R. J. Wood, C. J. Walsh, Advanced Materials Technologies, 2017, 2(9), p.1700136.

[53] A. T. Asbeck, S. M. De Rossi, K. G. Holt, C. J. Walsh, The International Journal of Robotics Research, 2015, 34(6), pp.744-762.

[54] L. N. Awad, J. Bae, K. O'donnell, S.M.De Rossi, K. Hendron, L. H. Sloot, P. Kudzia, S. Allen, K. G. Holt, T. D. Ellis, C. J. Walsh, Science Translational Medicine, 2017, 9(400).

[55] Y. Ding, M. Kim, S. Kuindersma, C. J. Walsh, Science Robotics, 2018, 3(15).

[56] B. T. Quinlivan, S. Lee, P. Malcolm, D. M. Rossi, M. Grimmer, C. Siviy, N. Karavas, D. Wagner, A. Asbeck, I. Galiana, C.J. Walsh, Science Robotics, 2017, 2(2), p.eaah4416.

[57] L. Stirling, C. H. Yu, J. Miller, E. Hawkes, R. Wood, E. Goldfield, R. Nagpal, Journal of Materials Engineering and Performance, 2011, 20(4-5), pp.658-662.

[58] Y. J. Lai, L.J. Yeh, M. C. Chiu, Sensors and Actuators A: Physical, 2012, 173(1), pp.210-218.

[59] A. Hadi, K. Alipour, S. Kazeminasab, M. Elahinia, Journal of Intelligent Material Systems and Structures, 2018, 29(8), pp.1575-1585.

[60] S. Kazeminasab, A. Hadi, K. Alipour, M. Elahinia, M., Industrial Robot, 2018, 45(5).

[61] M. Hillman, Advances in Rehabilitation Robotics, 2004, pp. 25-44. Springer, Berlin, Heidelberg.

[62] D. Serrano, D.S. Copaci, L. Moreno, D. Blanco, IEEE/RSJ International Conference on Intelligent Robots and Systems (IROS), 2018, pp. 2318-2323. IEEE.

[63] A. Villoslada, A. Flores, D. Copaci, D. Blanco, L. Moreno, L., Robotics and Autonomous Systems, 2015, 73, pp.91-101.

[64] D. Copaci, D. Blanco, L. Moreno, Proceedings of the Joint Workshop on Wearable Robotics and Assistive Devices, International Conference on Intelligent Robots and Systems, 2016, pp. 9-14, Daejeon, Korea.

[65] D. Copaci, A. Flores, F. Rueda, I. Alguacil, D. Blanco, L. Moreno, Converging Clinical and Engineering Research on Neurorehabilitation II. Biosystems \& Biorobotics, 2017, 15, pp. 477-481. Springer.

[66] K. K. Wu, Foot orthoses: principles and clinical applications, 1990, vol. 26, Williams \& Wilkins.

[67] M. G. Mataee, M. T. Andani, M. Elahinia, Journal of Intelligent Material Systems and Structures, 2015, 26(6), pp.639-651.

[68] F. Sadeghian, M. R. Zakerzadeh, M. Karimpour, M. Baghani, Journal of Intelligent Material Systems and Structures, 2018, 29(15), pp.3136-3150.

[69] C. T. Hau, D. Gouwanda, A. A. Gopalai, L.C. Yee, F.A.B. Hanapiah, 39th Annual International Conference of the IEEE Engineering in Medicine and Biology Society (EMBC) 2017, pp. 946-949. IEEE. 
[70] S. Pittaccio, L. Garavaglia, C. Ceriotti, F. Passaretti, Journal of functional biomaterials, 2015, 6(2), pp.328-344.

[71] P. Joudzadeh, A. Hadi, K. Alipour, 4th International Conference on Robotics and Mechatronics (ICROM), 2016, pp. 530-535. IEEE.

[72] S.J. Park, C.H. Park, Scientific Reports, 2019, 9(1), pp.1-8.

[73] L.J. Romasanta, M.A. López-Manchado, R. Verdejo, Progress in Polymer Science, 2015, 51, pp.188-211.

[74] M. Lidka, A.D. Price, A.L. Trejos, IEEE Canadian Conference on Electrical \& Computer Engineering (CCECE), 2018, pp. 1-4. IEEE.

[75] A. Behboodi, S.C.K. Lee, IEEE 16th International Conference on Rehabilitation Robotics (ICORR), 2019, pp. 499-505. IEEE.

[76] Compliant Transducer Systems GmbH, http://www.ct-systems.ch/.

[77] M. Duduta, E. Hajiesmaili, H. Zhao, R.J. Wood, D. R. Clarke, Proceedings of the National Academy of Sciences, 2019, 116(7), pp.2476-2481.

[78] F. Carpi, A. Mannini, D. De Rossi, Electroactive Polymer Actuators and Devices (EAPAD), 2008, 6927, p. 692705. International Society for Optics and Photonics.

[79] A. Behboodi, C. DeSantis, J. Lubsen, S.C.K. Lee, 42nd Annual International Conference of the IEEE Engineering in Medicine \& Biology Society (EMBC), 2020, pp. 4930-4935. IEEE.

[80] L. Saharan, A. Sharma, M.J. de Andrade, R.H. Baughman, Y. Tadesse, Active and Passive Smart Structures and Integrated Systems, 2017, Vol. 10164, p. 1016428. International Society for Optics and Photonics.

[81] H. Amin, S.F. Assal, IEEE International Conference on Mechatronics and Automation (ICMA), 2018, pp. 997-1002. IEEE.

[82] G. Kofod, Journal of Physics D: Applied Physics, 2008, 41(21), p.215405.

[83] Y. Li, M. Hashimoto, Smart Materials and Structures, 2017, 26(12), p.125003.

[84] S. Pourazadi, S. Ahmadi, C. Menon, Smart Materials and Structures, 2014, 23(6), p.065007.

[85] E.Y. Kramarenko, A.V. Chertovich, G.V. Stepanov, A.S. Semisalova, L.A. Makarova, N.S. Perov, A.R. Khokhlov, Smart Materials and Structures, 2015, 24(3), p.035002.

[86] G.V. Stepanov, E.Y. Kramarenko, D.A. Semerenko, Journal of Physics: Conference Series, 2013, 412(1), p. 012031. IOP Publishing.

[87] L.A. Makarova, T.A. Nadzharyan, Y.A. Alekhina, G.V. Stepanov, E.G. Kazimirova, N.S. Perov, E.Y. Kramarenko, Smart Materials and Structures, 2017, 26(9), p.095054.

[88] T.A. Nadzharyan, L.A. Makarova, E.G. Kazimirova, N.S. Perov, E.Y. Kramarenko, 2018. Influence of the geometry on magnetic interactions in a retina fixator based on a magnetoactive elastomer seal. Journal of Physics: Conference Series, 2018, 994(1), p.012002.

[89] Y.A. Alekhina, L.A. Makarova, S.A. Kostrov, G.V. Stepanov, E.G. Kazimirova, N.S. Perov, E.Y. Kramarenko, 2019. Journal of Applied Polymer Science, 2019, 136(17), p.47425.

[90] C. Ohm, M. Brehmer, R. Zentel, Advanced Materials, 2010, 22(31), pp.3366-3387.

[91] C. Ferrantini, J.M. Pioner, D. Martella, R. Coppini, N. Piroddi, P. Paoli, M. Calamai, F.S. Pavone, D.S. Wiersma, C. Tesi, E. Cerbai, Circulation research, 2019, 124(8), pp. e44-e54.

[92] M. Asadnia, A.G.P. Kottapalli, J.M. Miao, M.S. Triantafyllou, 28th IEEE International Conference on Micro Electro Mechanical Systems (MEMS), 2015, pp. 678-681. IEEE. 
[93] N.R. Alluri, S. Selvarajan, A. Chandrasekhar, B. Saravanakumar, J.H. Jeong, S.J. Kim, 2017. Composites Science and Technology, 2017, 142, pp.65-78.

[94] K.A. Ahmad, M.F.A. Rahman, R. Boudville, A.A. Manaf, N. Abdullah, 12th International Conference on Sensing Technology (ICST), 2018, pp. 149-153. IEEE.

[95] S. Rajala, R. Mattila, I. Kaartinen, J. Lekkala, IEEE Sensors Journal, 2017, 17(20), pp.6798-6805. IEEE.

[96] W. Ma, X. Zhang, G. Yin, 13th International Conference on Ubiquitous Robots and Ambient Intelligence (URAI), 2016, pp. 587-592. IEEE.

[97] M. Hosono, S. Ino, M. Sato, K. Yamashita, T. Izumi. T. Ifukube, Proceedings of the 3rd Asia International Symposium on 0echatronics,2008, p. 473.

[98] S. Ino, M. Hosono, M. Sato, S. Nakajima, K. Yamashita, T. Izumi, World Congress on Medical Physics and Biomedical Engineering, Munich, Germany, 2009, pp. 287-290, Springer, Berlin, Heidelberg.

[99] S. Ino, M. Sato, M. Hosono, T. Izumi, Sensors and Actuators B: Chemical, 2009, 136(1), pp.86-91. 